\title{
Bitcoin and Cryptocurrencies - Not for the Faint-Hearted
}

\author{
Jörg Osterrieder \\ School of Engineering, Zurich University of Applied Sciences, Winterthur, Switzerland \\ Tel: 41-0-58-934-4594Ｅ-mail: joerg.osterrieder@zhaw.ch
}

Julian Lorenz

E-mail: research@algotradingstrategies.com

\begin{abstract}
Martin Strika
School of Engineering, Zurich University of Applied Sciences, Winterthur, Switzerland E-mail:martin.strika@gmx.ch
\end{abstract}

Received: Dec. 12, 2016 Accepted: Dec. 22, 2016 Published: Jan. 23, 2017

doi:10.5296/ifb.v4i1.10451 URL: http://dx.doi.org/10.5296/ifb.v4i1.10451

\begin{abstract}
Cryptocurrencies became popular with the emergence of Bitcoin and have shown an unprecedented growth over the last few years. As of November 2016, more than 720 cryptocurrencies exist, with Bitcoin still being the most popular one. We provide both a statistical analysis as well as an extreme value analysis of the returns of the most important cryptocurrencies. A particular focus is on the tail risk characteristics and we will provide an in-depth univariate and multivariate extreme value analysis. The tail dependence of cryptocurrencies is investigated (using both empirical and Gaussian copulas). For investors - especially institutional ones - as well as regulators, an understanding of the risk and tail characteristics are of utmost importance. For cryptocurrencies to become a mainstream investable asset class, studying these properties is necessary. Our findings show that cryptocurrencies exhibit strong non-normal characteristics, large tail dependencies, depending on the particular cryptocurrencies and heavy tails. Statistical similarities can be observed for cryptocurrencies that share the same underlying technology. This has implications for risk management, financial engineering (such as derivatives on cryptocurrencies) — both from an investor's as well as from a regulator's point of view. To our
\end{abstract}


knowledge, this is the first detailed study looking at the extreme value behaviour of cryptocurrencies, their correlations and tail dependencies as well as their statistical properties.

Keywords: Bitcoin, Cryptocurrency, Extreme value theory, Risk management, Extreme events 


\section{Introduction and Motivation}

Since Bitcoin became the first decentralized cryptocurrency in 2009, numerous cryptocurrencies have been created. Bitcoin, as of November 2016, is the largest of its kind in terms of total market value, representing over $81 \%$ of the total market of cryptocurrencies (CoinMarketCap, 2016). The second and third largest cryptocurrencies are Ethereum and Ripple, representing $7.6 \%$ and $2.4 \%$ of the market. There are more than 720 cryptocurrencies with the top ten of them (Bitcoin, Ethereum, Ripple, Litecoin, Ethereum Classic, Monero, Dash, Augur, MaidSafeCoin, Waves) representing about 95\% of the market (CoinMarketCap, 2016).

A cryptocurrency is a digital asset designed to work as a medium of exchange using cryptography to secure the transactions and to control the creation of additional units of the currency. Cryptocurrencies are a subset of alternative currencies, or specifically of digital currencies.

Regulators need to ask themselves if cryptocurrencies can freely be used by retail customers. We will show that the risks of cryptocurrencies are larger than any other traditional investment or currency that is available to retail clients.

The market capitalization of the top ten cryptocurrencies as of November 8, 2016 can be seen in the following Table 1 (see CoinMarketCap, 2016):

Table 1. Market capitalization of cryptocurrencies

\begin{tabular}{|l|l|l|}
\hline Name & Market cap (bn USD) & Market cap, relative \\
\hline Bitcoin & 11350 & $83.4 \%$ \\
\hline Ethereum & 932 & $6.8 \%$ \\
\hline Ripple & 293 & $2.2 \%$ \\
\hline Litecoin & 185 & $1.4 \%$ \\
\hline Monero & 89 & $0.7 \%$ \\
\hline Ethereum Classic & 80 & $0.6 \%$ \\
\hline Dash & 67 & $0.5 \%$ \\
\hline Augur & 53 & $0.4 \%$ \\
\hline Nem & 37 & $0.3 \%$ \\
\hline MaidSafeCoin & 36 & $0.3 \%$ \\
\hline
\end{tabular}

The main purpose of the paper is to understand and characterize cryptocurrency exchange rates from a statistical point of view. The underlying technological issues are well-known, however the actual behavior of returns is not yet well understood. We are therefore describing the statistical properties of cryptocurrencies and we focus in particular on extreme value characteristics. The analysis of extreme events is particularly relevant if you are an investor into cryptocurrencies and you want to understand what risks you are facing. For a 
characterization of the parametric distributions of cryptocurrencies, see Osterrieder (2016). In Osterrieder \& Lorenz (2016), the authors analyze the Bitcoin exchange rates and compare them to the G10 currencies. For the analysis fiat currencies, Nadarajah et al. (2015) model exchange rate returns and consider the question, what flexible distribution to use.

The paper is organized as follows. In section 2, we give an overview of the data which we used and the sources from which it was retrieved. Section 3 looks at statistical properties of cryptocurrencies, computes volatilities and correlations. In Section 4 we will use concepts and results from extreme value theory to analyze the behaviour of cryptocurrencies during extreme events with a particular focus on the left tail of the distribution, implicitly assuming that we own cryptocurrencies. We use copula theory to look at the dependence between different currencies and compute the tail dependence coefficients as well as the extremal index. Furthermore, two important risk measures, value-at-risk and expected shortfall are computed. A closer look at the generalized Pareto distribution and the generalized extreme value distribution will be made in section 5. The last section 6 concludes and summarizes our findings.

\subsection{What are Cryptocurrencies?}

A cryptocurrency is a digital asset designed to work as a medium of exchange that is created and managed through the use of advanced encryption techniques known as cryptography. Cryptocurrencies use decentralized control as opposed to centralized electronic money/centralized banking systems. The decentralized control is related to the use of bitcoin's blockchain transaction database in the role of a distributed ledger.

\subsection{A Short Description of the Individual Cryptocurrencies}

In our study, we analyze six out of the ten largest cryptocurrencies. The selected currencies are: Bitcoin, Litecoin, Ripple, Monero, MaidSafeCoin, Dash. We have chosen those six because they have existed on or before June 2014, so that we have sufficient data to analyze the returns.

Bitcoin (BTC) is a decentralized currency that uses peer-to-peer technology, which enables all functions such as currency issuance, transaction processing and verification to be carried out collectively by the network. While this decentralization renders Bitcoin free from government manipulation or interference, the flipside is that there is no central authority to ensure that things run smoothly or to back the value of a Bitcoin. Bitcoins are created digitally through a "mining" process that requires powerful computers to solve complex algorithms and crunch numbers. These characteristics make Bitcoin fundamentally different from a fiat currency, which is backed by the full faith and credit of its government. Bitcoin is designed around the idea of using cryptography to control the creation and transfer of money, rather than relying on central authorities.

Litecoin (LTC) is regarded as Bitcoin's leading rival at present, and it is designed for processing smaller transactions faster. It was founded in October 2011. It is a peer-to-peer Internet currency based on the Bitcoin protocol but differs from Bitcoin in that it can be efficiently mined with consumer-grade hardware. Litecoin provides faster transaction 
confirmations and uses a memory-hard, scrypt-based mining proof-of-work algorithm to target the regular computers and GPUs most people already have. One of the aims of Litecoin was to provide a mining algorithm that could run at the same time, on the same hardware used to mine bitcoins.

Dash (formerly known as Darkcoin and XCoin) is an open source peer-to-peer cryptocurrency that offers instant transactions, private transactions and token fungibility. Dash operates a decentralized governance and budgeting system, making it the first decentralized autonomous organization. Dash uses a chained hashing algorithm called X11 for the proof-of-work. Instead of using the SHA-256 (from well-known Secure Hash Algorithm family) or scrypt it uses 11 rounds of different hashing functions.

Monero (XMR) is an open source cryptocurrency created in April 2014 that is focused on privacy, decentralisation and scalability. Unlike many cryptocurrencies that are derivatives of Bitcoin, Monero is based on the CryptoNote protocol and possesses significant algorithmic differences relating to blockchain obfuscation.

Ripple (XRP) was launched by OpenCoin, a company founded by technology entrepreneur Chris Larsen in 2012. Like Bitcoin, Ripple is both a currency and a payment system. The currency component is XRP, which has a mathematical foundation like Bitcoin. Ripple is one of the main contestants for the title of Bitcoin successor. It uses a consensus algorithm by utilizing collectively-trusted subnetworks within the larger network.

MaidSafeCoin (Safecoin) is a digital cryptocurrency token that is in the heart of the SAFE Network. Safecoins are being distributed entirely automated with no human intervention by an algorithm. Only 4.3 billion MaidSafeCoins will ever be in circulation at one time and each coin will have its own unique identity. Furthermore Safecoins will be recycled when they are being exchanged for network services, so there will be a new supply of coins for users to earn. Safecoins are given to users in return for them providing some of their unused computing resources to the network. These include free storage space, processing power, and Internet connectivity, this process of providing your resources and earning Safecoin in return on the platform is referred to as farming.

\section{Data}

We are using historical global price indices for cryptocurrencies from the database BNC2 from quandl.com, which shows aggregated cryptocurrency prices from multiple exchanges providing a weighted average cryptocurrency price. They regularly collect information from different online exchanges, collate it and calculate a weighted average price for the different cryptocurrencies based on activity, trading volume, liquidity and other factors. Daily data is downloaded from June 23, 2014 until the end of September 2016. On purpose, we have chosen to start only in June 2014, because that allows us to analyze six out of the top ten cryptocurrencies by market capitalization as of November 2016.

The top ten of them (Bitcoin, Ethereum, Ripple, Litecoin, Ethereum Classic, Monero, Dash, Augur, MaidSafeCoin, NEM) represent more than $95 \%$ of the market capitalization. The six cryptocurrencies that we have chosen out of the top ten are: Bitcoin, Dash, LiteCoin, 


\section{Macrothink}

MaidSafeCoin, Monero and Ripple. We have omitted Ethereum, with an initial release in July 30, 2015, Ethereum Classic, which only started trading in 2016, Augur and NEM, which also only came into existence in 2015 . In total, our choice of cryptocurrencies represents $88 \%$ of the market capitalization as of November 2016.

\section{Statistical Properties of Cryptocurrencies}

Before we go into the extreme value behaviour of cryptocurrencies, we analyze the statistical properties of cryptocurrencies based on their returns.

\subsection{Distribution of Returns}

We want to show the distribution of returns and compare them to a normal distribution. Deviations of the cryptocurrency distribution from the normal distribution will justify our choice of using extreme value theory to describe the tails. In Figure 1, we are showing the histogram of daily returns of the Bitcoin / USD exchange rate. The red line which is overlaid, shows a normal distribution with mean and standard deviation taken from the empirical Bitcoin/ USD exchange rates. The blue line is showing a Gaussian kernel-density estimator with bandwidth multiple 0.5 . We see a substantial deviation from the normal distribution.

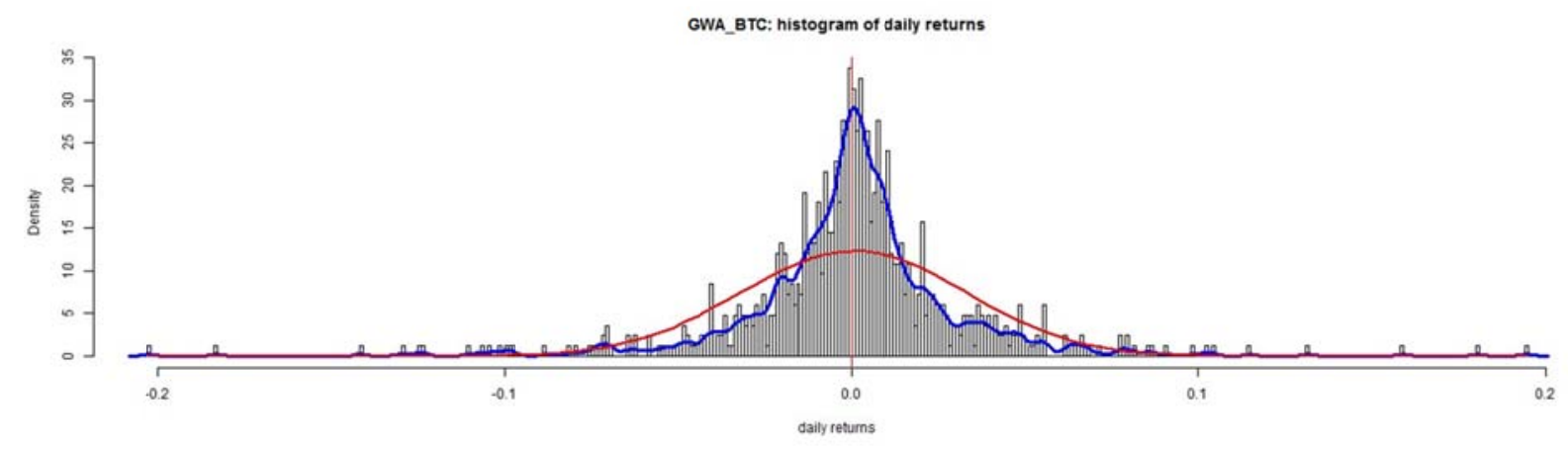

Figure 1. Histogram of Bitcoin/USD exchange rate with fitted normal distribution and Gaussian KDE overlaid

The next Figure 2 shows the qq-plot of the empirical Bitcoin returns versus the quantiles of the standard normal distribution. Again, we observe large deviations from the normal distribution both on the left and the right tail. 


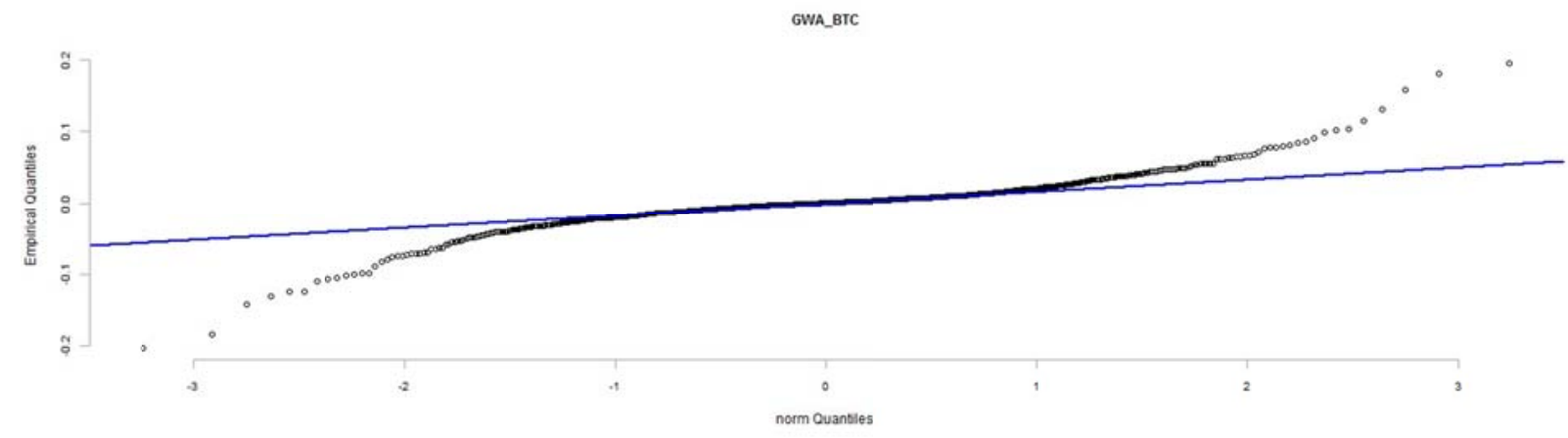

Figure 2. QQ-Plot of the Bitcoin/USD exchange rate versus the normal distribution

The following five Figures 3, 4, 5, 6, 7 show the empirical histogram of our five other cryptocurrencies, Dash, Litecoin, MaidSafeCoin, Ripple and Monero. We have chosen to restrict the $\mathrm{x}$-axis to returns between -0.2 and 0.2 , thus omitting occasional large outliers.

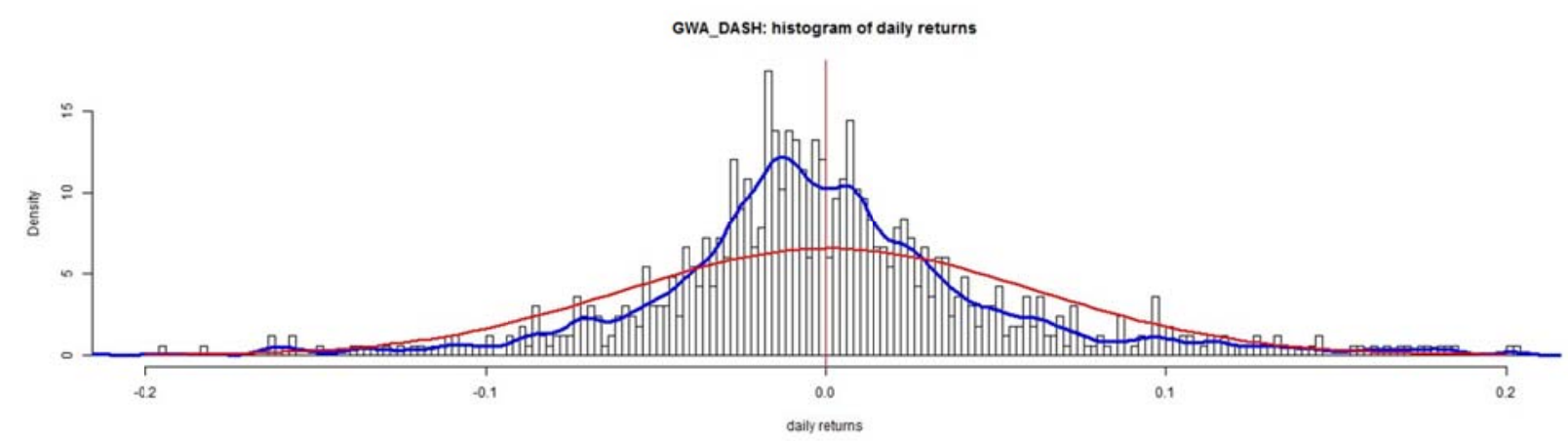

Figure 3. Histogram of Dash/USD exchange rate with fitted normal distribution and Gaussian KDEoverlaid

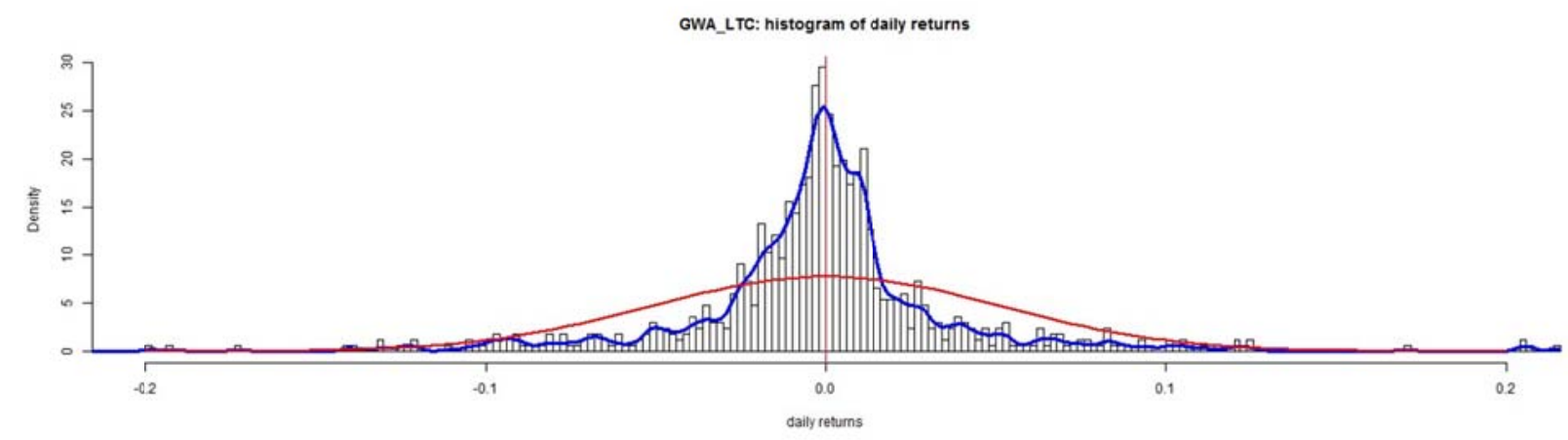

Figure 4. Histogram of LTC/USD exchange rate with fitted normal distribution and Gaussian KDE overlaid 


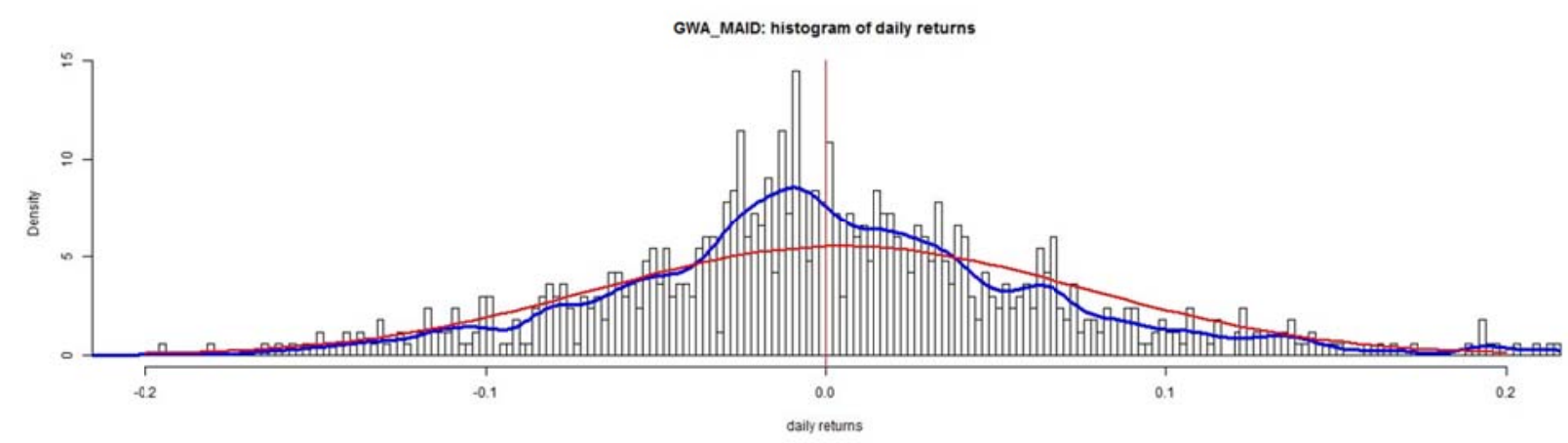

Figure 5. Histogram of MAID/USD exchange rate with fitted normal distribution and Gaussian KDEoverlaid

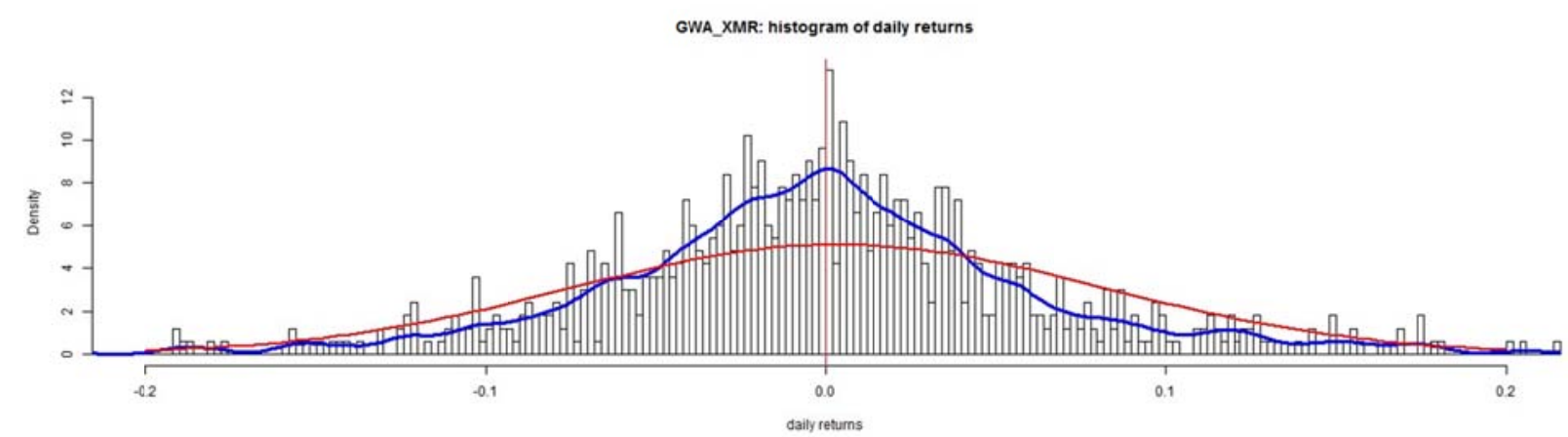

Figure 6. Histogram of XMR/USD exchange rate with fitted normal distribution and Gaussian KDEoverlaid

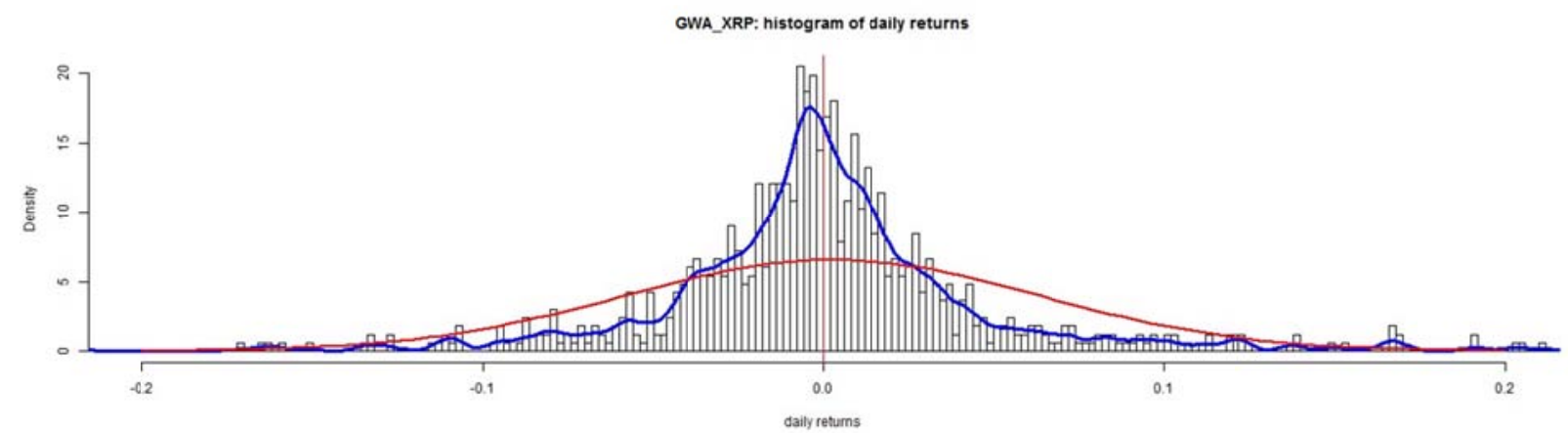

Figure 7. Histogram of XRP/USD exchange rate with fitted normal distribution and Gaussian KDEoverlaid

We see a substantial deviation from the normal distribution in all six plots. In the following five charts, $8,9,10,11,12$ we show the qq-plot of the empirical returns versus the quantiles of the standard normal distribution. Again, we observe large deviations from the normal distribution both on the left and the right tail for all cryptocurrencies. 


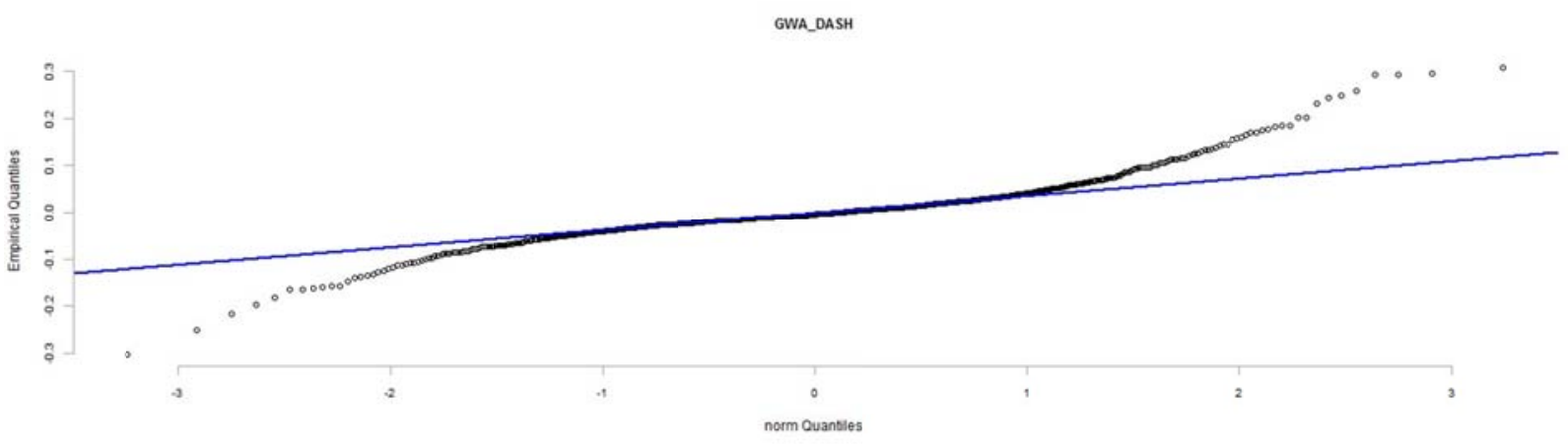

Figure 8. QQ-Plot of the DASH/USD exchange rate versus the normal distribution

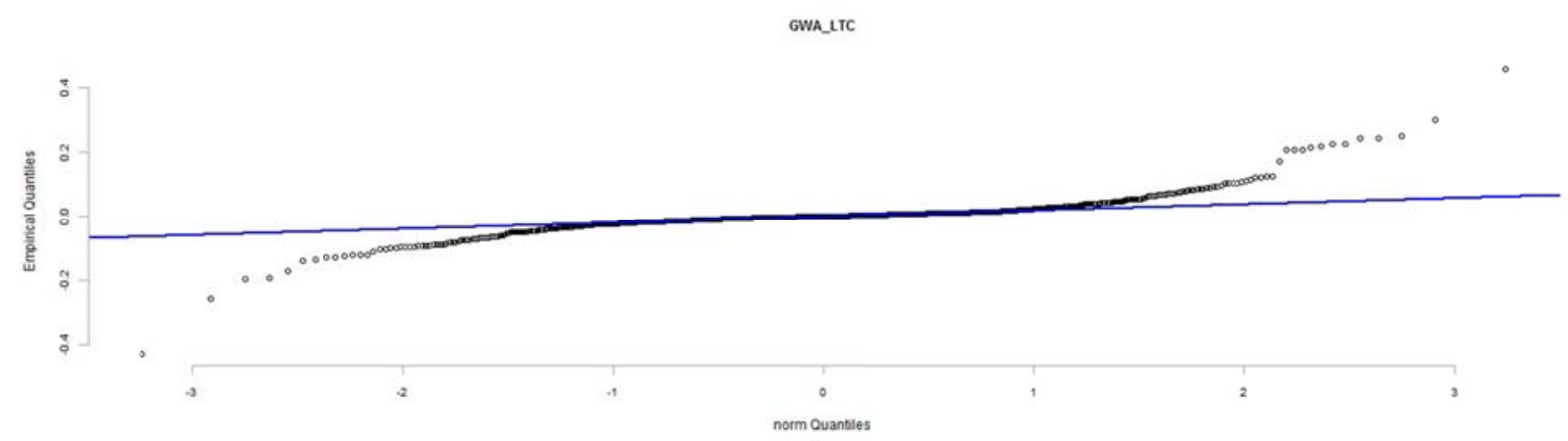

Figure 9. QQ-Plot of the LTC/USD exchange rate versus the normal distribution

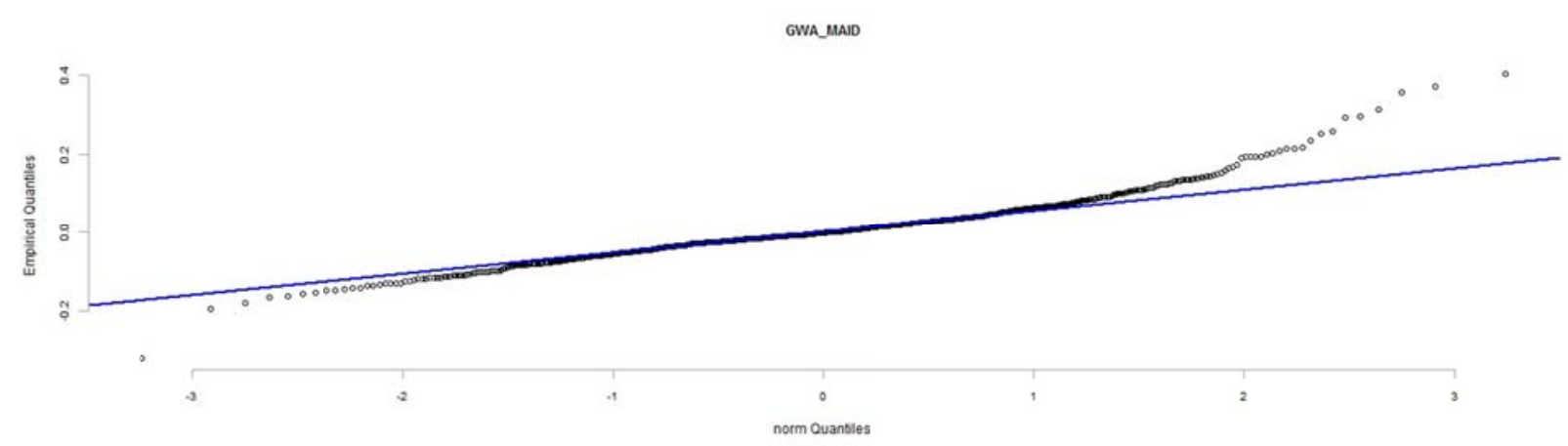

Figure 10. QQ-Plot of the MAID/USD exchange rate versus the normal distribution 


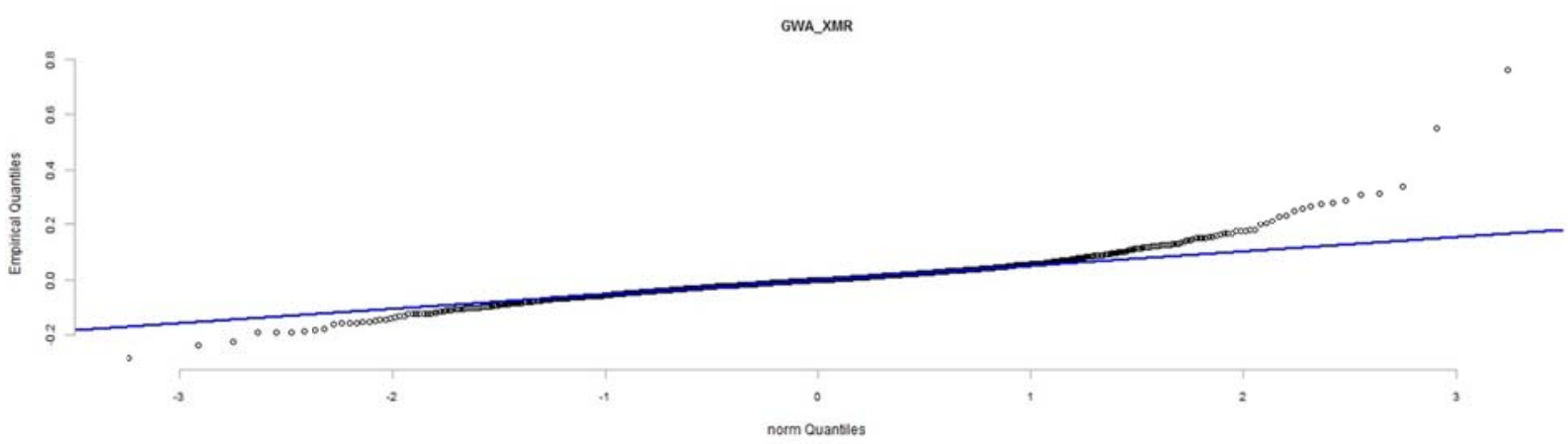

Figure 11. QQ-Plot of the XMR/USD exchange rate versus the normal distribution

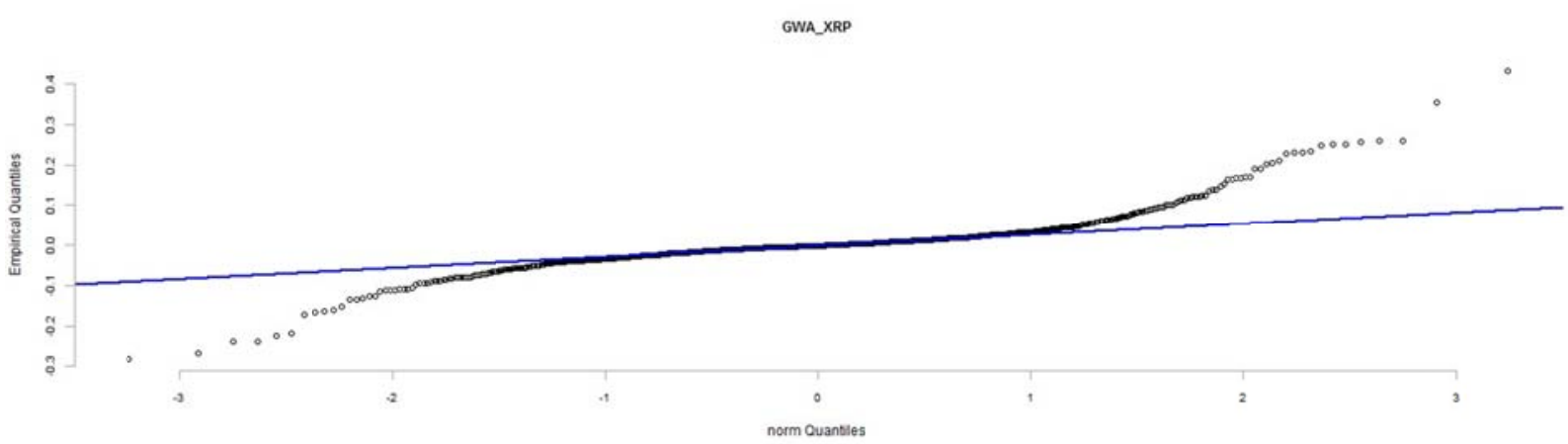

Figure 12. QQ-Plot of the XRP/USD exchange rate versus the normal distribution

\subsection{Volatility}

We are recording here the volatilities of our cryptocurrencies from June 2014 until the end of September 2016. The annualized standard deviation of returns over this period can be seen in the following Table 2:

Table 2. Annualized volatility

\begin{tabular}{|l|l|}
\hline Exchange rate & Annualized Volatility \\
\hline Bitcoin/USD & $62 \%$ \\
\hline Dash/USD & $116 \%$ \\
\hline Litecoin/USD & $97 \%$ \\
\hline Maid/USD & $138 \%$ \\
\hline Monero/USD & $149 \%$ \\
\hline Ripple/USD & $116 \%$ \\
\hline
\end{tabular}

In the following Figure 13, we have plotted the 90-day rolling volatility (annualized) for our cryptocurrencies versus the USD. 


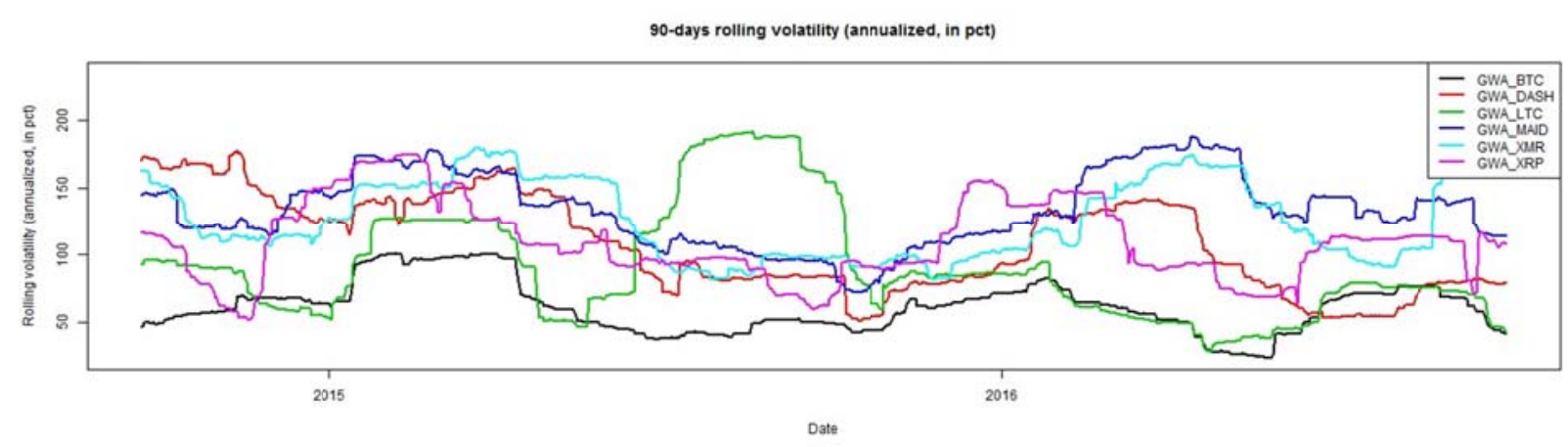

Figure 13. 90-days rolling volatility (annualized, in pct)

We observe that cryptocurrencies have enormous volatility which is substantially larger than any of the established fiat currencies or any of the standard financial assets.

\subsection{Correlations}

An interesting question regarding cryptocurrencies is how their multivariate statistical properties look like, and we start by studying cryptocurrency correlation in the forms of Pearson's correlation coefficient $\rho$, Spearman's rank correlation coefficient and Kendall's tau correlation coefficient (for definitions and comparisons of these three correlation statistics, see for instance (Chok, 2010) and (Sheskin, 2007). Tables 3, 4 and 5 summarize the correlation of the six cryptocurrencies (versus USD) in our study over our full dataset (2014/06 to 2016/09).

Table 3. Kendall correlation for cryptocurrencies

\begin{tabular}{|l|l|l|l|l|l|l|}
\hline & Bitcoin & Dash & Litecoin & Maid & Monero & Ripple \\
\hline Bitcoin & 1 & 0.255 & 0.584 & 0.192 & 0.242 & 0.125 \\
\hline Dash & 0.255 & 1 & 0.214 & 0.142 & 0.145 & 0.116 \\
\hline Litecoin & 0.584 & 0.214 & 1 & 0.126 & 0.180 & 0.130 \\
\hline MAID & 0.192 & 0.142 & 0.126 & 1 & 0.147 & 0.083 \\
\hline Monero & 0.242 & 0.145 & 0.180 & 0.147 & 1 & 0.054 \\
\hline Ripple & 0.125 & 0.116 & 0.130 & 0.083 & 0.054 & 1 \\
\hline
\end{tabular}

Table 4. Spearman correlation for cryptocurrencies

\begin{tabular}{|l|l|l|l|l|l|l|}
\hline & Bitcoin & Dash & Litecoin & Maid & Monero & Ripple \\
\hline Bitcoin & 1 & 0.362 & 0.753 & 0.278 & 0.351 & 0.183 \\
\hline Dash & 0.362 & 1 & 0.311 & 0.208 & 0.208 & 0.172 \\
\hline Litecoin & 0.753 & 0.311 & 1 & 0.186 & 0.263 & 0.186 \\
\hline MAID & 0.278 & 0.208 & 0.186 & 1 & 0.211 & 0.122 \\
\hline Monero & 0.351 & 0.208 & 0.263 & 0.211 & 1 & 0.079 \\
\hline Ripple & 0.183 & 0.172 & 0.186 & 0.122 & 0.079 & 1 \\
\hline
\end{tabular}




\section{Macrothink}

Table 5. Pearson correlation for cryptocurrencies

\begin{tabular}{|l|l|l|l|l|l|l|}
\hline & Bitcoin & Dash & Litecoin & Maid & Monero & Ripple \\
\hline Bitcoin & 1 & 0.393 & 0.611 & 0.337 & 0.353 & 0.152 \\
\hline Dash & 0.393 & 1 & 0.248 & 0.194 & 0.175 & 0.110 \\
\hline Litecoin & 0.611 & 0.248 & 1 & 0.214 & 0.212 & 0.103 \\
\hline MAID & 0.337 & 0.194 & 0.214 & 1 & 0.165 & 0.089 \\
\hline Monero & 0.353 & 0.175 & 0.212 & 0.165 & 1 & 0.047 \\
\hline Ripple & 0.152 & 0.110 & 0.103 & 0.089 & 0.047 & 1 \\
\hline
\end{tabular}

Overall, the levels of correlation are mixed and somewhat modest—with Bitcoin and Litecoin showing the highest correlation (Pearson's correlation coefficient of approximately 0.6).

Here, the technical similarities between Bitcoin and Litecoin are also clearly reflected in their statistical properties.

Looking at the time-evolution of correlations, a more complex picture emerges. Figure 14 shows the rolling 90 day correlation of Bitcoin (BTC) to the other 5 cryptocurrencies. As can be seen, correlations vary substantially over time.

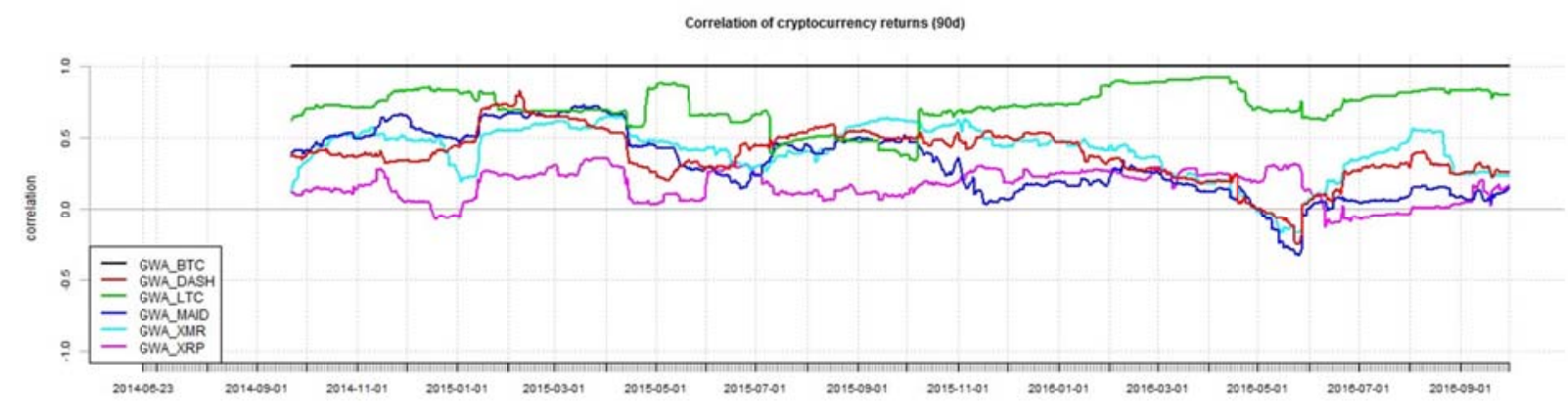

Figure 14. 90-days rolling correlation

(Gandal \& Halaburda, 2016) have attributed this time-changing correlation in the crypto-currency space to two opposing effects (albeit investigating a different time period prior to ours): reinforcement (one or more specific cryptocurrencies outperforming in a winner-take-all race against other cryptocurrencies, leading to negative correlation) and substitution (cryptocurrencies treated as financial assets - popularized by Bitcoin-, in which people seek alternative investments as one cryptocurrency gets too expensive-leading to positive correlation as cryptocurrencies collectively gain in market value, or collectively drop if overall sentiment for cryptocurrencies is losing ground). This is due to the fact that the various cryptocurrencies are to a large extent similar in technology and functionality, but provide alternatives to Bitcoin by aiming to fix what their developers thought to be 
shortcomings of the original Bitcoin protocol (for instance, slow transaction times or the incentive structure of mining new coins in the network).

In our study period, we can see the Bitcoin/Litecoin correlation being rather high and somewhat stable-whereas the correlation of Bitcoin to the other four crypto-currencies is dropping in the second half, especially in 2016. This points to possibly mild substitution effects between Bitcoin and Litecoin, and reinforcement in favor of these two versus the other four: Litecoin seems to be perceived as the most viable substitute, while the other cryptocurrencies are less correlated to Bitcoin - and even negatively correlated at times, pointing towards these cryptocurrencies giving way to the other two.

\section{Extreme Value Analysis of Cryptocurrencies}

Apart from looking at the properties of the cryptocurrency returns over the entire spectrum, we also want to analyze extreme events. Large losses or gains are particularly relevant for risk management and investment purposes. Furthermore, when considering an investment in multiple cryptocurrencies, it is important to consider their correlation, common moves as well as the dependence of returns in extreme scenarios. We will use techniques and tools from extreme value theory to analyse the correlation of cryptocurreny returns as well their behaviour under tail events.

\subsection{Copulas}

Copulas allows one to focus on the dependence of random variables by separating the dependence from the univariate distributions. An $n$-dimensional joint distribution can be decomposed into its $n$ univariate marginal distributions and an $n$-dimensional copula. To be specific, let $Y=\left(Y_{1}, \ldots, Y_{n}\right)^{T}$ be a random vector with cumulative distribution function $F$ and, for let $F_{i}$ denote the marginal distribution of $Y_{i}$. Then there exists a copula such that, for all $y=\left(y_{1}, \ldots, y_{n}\right) \in \mathbb{R}^{n}$,

$$
F(y)=C\left(F_{1}\left(y_{1}\right), \ldots, F_{n}\left(y_{n}\right)\right)
$$

A copula $C$ of the random vector $Y$ is thus a function that maps the univariate marginal distributions $F_{1}, \ldots, F_{n}$ to the joint distribution $F$, and we write $Y \sim F=C\left(F_{1}, \ldots, F_{n}\right)$. For continuous random variables, the copula is unique. It is the joint distribution of the probability integral transforms of the original variables, which are defined as $U_{i}=F_{i}\left(Y_{i}\right)$. Then $U=\left(U_{1}, \ldots, U_{n}\right)^{T} \sim C$. It is well known that if $Y_{i}$ is continuous, then $U_{i} \sim U(0,1)$ and in this case a copula is any joint distribution function with $U(0,1)$ margins. What makes the representation in (1) particularly useful for applied researchers is the converse of Sklar's theorem: given any collection of univariate distributions $F_{1}, \ldots, F_{n}$ and any copula $C$, the function $F$ defined by (1) above defines a valid joint distribution with marginal distributions $F_{1}, \ldots, F_{n}$. For example, one might combine a normally distributed variable with an exponentially distributed variable via a Clayton copula, and obtain an unusual but valid bivariate distribution. The ability to combine marginal distributions with a copula model allows the researcher to directly draw on the large body of research on modeling univariate distributions, leaving only the task of modeling the dependence structure. 


\section{Ml Macrothink}

Definition 1 (Copulas). In probabilistic terms, $C:[0,1]^{d} \rightarrow[0,1]$ is a d-dimensional copula if $C$ is a joint cumulative distribution function of a $d$-dimensional random vector on the unit cube $[0,1]^{d}$ with uniform marginals. In analytic terms, $C:[0,1]^{d} \rightarrow[0,1]$ is a d-dimensional copula if

- $C\left(u_{1}, \ldots, u_{i-1}, 0, u_{i+1}, \ldots, u_{d}\right)=0$, the copula is zero if one of the arguments is zero

- $C(1, \ldots, 1, u, 1, \ldots, 1)=u$, the copula is equal to $\mathrm{u}$ if one argument is $\mathrm{u}$ and all others 1 ,

- $C$ is $d$-non-decreasing, i.e., for each hyperrectangle $B=\prod_{i=1}^{d}\left[x_{i}, y_{i}\right] \subseteq[0,1]^{d}$ the

$C$-volume of $B$ is non-negative:

$$
\int_{B} d C(u)=\sum_{z \in \times_{i=1}^{d}\left\{x_{i}, y_{i}\right\}}(-1)^{N(z)} C(z) \geq 0,
$$

where the $N(z)=\#\left\{k: z_{k}=x_{k}\right\}$.

For instance, in the bivariate case, $C:[0,1] \times[0,1] \rightarrow[0,1]$ is a bivariate copula if $C(0, u)=C(u, 0)=0, C(1, u)=C(u, 1)=u$ and $C\left(u_{2}, v_{2}\right)-C\left(u_{2}, v_{1}\right)-C\left(u_{1}, v_{2}\right)+$ $C\left(u_{1}, v_{1}\right) \geq 0$ for all $0 \leq u_{1} \leq u_{2} \leq 1$ and $0 \leq v_{1} \leq v_{2} \leq 1$.

Copulas are useful in portfolio and risk management and help us analyse the effects of downside regimes by allowing the modelling of the marginals and dependence structure of a multivariate probability model separately.

In risk/portfolio management, copulas are used to perform stress-tests and robustness checks that are especially important during "downside/crisis/panic regimes" where extreme downside events may occur (e.g., the global financial crisis of 2007-2008).

As far as derivatives pricing is concerned, dependence modelling with copula functions is widely used in applications of financial risk assessment and actuarial analysis - for example in the pricing of collateralized debt obligations (CDOs).

\subsubsection{Empirical Copula}

We start our copula analysis by looking at the sample data and constructing the underlying copula.

Definition 2 (Empirical copula). When studying multivariate data, one might want to investigate the underlying copula. Suppose we have observations $\left(\mathrm{X}_{1}^{\mathrm{i}}, \mathrm{X}_{2}^{\mathrm{i}}, \ldots, \mathrm{X}_{\mathrm{d}}^{\mathrm{i}}\right), \mathrm{i}=1, \ldots, \mathrm{n}$, from a random vector $\left(\mathrm{X}_{1}, \mathrm{X}_{2}, \ldots, \mathrm{X}_{\mathrm{d}}\right)$ with continuous margins. The corresponding "true" copula observations would be $\left(\mathrm{U}_{1}^{\mathrm{i}}, \mathrm{U}_{2}^{\mathrm{i}}, \ldots, \mathrm{U}_{\mathrm{d}}^{\mathrm{i}}\right)=\left(\left(\mathrm{F}_{1}\left(\mathrm{X}_{1}^{\mathrm{i}}\right), \mathrm{F}_{2}\left(\mathrm{X}_{2}^{\mathrm{i}}\right), \ldots, \mathrm{F}_{\mathrm{d}}\left(\mathrm{X}_{\mathrm{d}}^{\mathrm{i}}\right)\right), \mathrm{i}=1, \ldots, \mathrm{n}\right.$.

However, the marginal distribution functions $F_{i}$ are usually not known. Therefore, one can construct pseudo copula observations by using the empirical distribution functions $F_{k}^{n}(x)=\frac{1}{n} \sum_{i=1}^{n} \mathbf{1}\left(X_{k}^{i} \leq x\right)$ instead. Then, the pseudo copula observations are defined as 


\section{Macrothink}

$\left(\widetilde{\mathrm{U}}_{1}^{\mathrm{i}}, \widetilde{\mathrm{U}}_{2}^{\mathrm{i}}, \ldots, \widetilde{\mathrm{U}}_{\mathrm{d}}^{\mathrm{i}}\right)=\left(\mathrm{F}_{1}^{\mathrm{n}}\left(\mathrm{X}_{1}^{\mathrm{i}}\right), \mathrm{F}_{2}^{\mathrm{n}}\left(\mathrm{X}_{2}^{\mathrm{i}}\right), \ldots, \mathrm{F}_{\mathrm{d}}^{\mathrm{n}}\left(\mathrm{X}_{\mathrm{d}}^{\mathrm{i}}\right)\right), \mathrm{i}=1, \ldots, \mathrm{n}$. The corresponding empirical copula is then defined as $\mathrm{C}^{\mathrm{n}}\left(\mathrm{u}_{1}, \ldots, \mathrm{u}_{\mathrm{d}}\right)=\frac{1}{\mathrm{n}} \sum_{\mathrm{i}=1}^{\mathrm{n}} \mathbf{1}\left(\widetilde{\mathrm{U}}_{1}^{\mathrm{i}} \leq \mathrm{u}_{1}, \ldots, \widetilde{\mathrm{U}}_{\mathrm{d}}^{\mathrm{i}} \leq \mathrm{u}_{\mathrm{d}}\right)$. The components of the pseudo copula samples can also be written as $\widetilde{U}_{k}^{i}=R_{k}^{i} / n, R_{k}^{i}$ is the rank of the observation $\mathrm{X}_{\mathrm{k}}^{\mathrm{i}}: \mathrm{R}_{\mathrm{k}}^{\mathrm{i}}=\sum_{\mathrm{j}=1}^{\mathrm{n}} \mathbf{1}\left(\mathrm{X}_{\mathrm{k}}^{\mathrm{j}} \leq \mathrm{X}_{\mathrm{k}}^{\mathrm{i}}\right)$. Therefore, the empirical copula can be seen as the empirical distribution of the rank transformed data.

One of the most important theorems in copula theory is Sklar's theorem.

Theorem 1 (Sklar). Sklar's theorem named after Abe Sklar, provides the theoretical foundation for the application of copulas. Sklar's theorem states that every multivariate cumulative distribution function $H\left(x_{1}, \ldots, x_{d}\right)=\mathbb{P}\left[X_{1} \leq x_{1}, \ldots, X_{d} \leq x_{d}\right]$ of a random vector $\left(X_{1}, X_{2}, \ldots, X_{d}\right)$ can be expressed in terms of its marginals $F_{i}(x)=\mathbb{P}\left[X_{i} \leq x\right]$ and a copula $C$. Indeed: $H\left(x_{1} \ldots, x_{d}\right)=C\left(F_{1}\left(x_{1}\right), \ldots, F_{d}\left(x_{d}\right)\right)$. In case that the multivariate distribution has a density $h$, and this is available, it holds further that $h\left(x_{1}, \ldots, x_{d}\right)=$ $c\left(F_{1}\left(x_{1}\right), \ldots, F_{d}\left(x_{d}\right)\right) \cdot f_{1}\left(x_{1}\right) \cdot \ldots \cdot f_{d}\left(x_{d}\right)$, where $c$ is the density of the copula. The theorem also states that, given $H$, the copula is unique on $\operatorname{Ran}\left(F_{1}\right) \times \cdots \times \operatorname{Ran}\left(F_{d}\right)$, which is the Cartesian product of the ranges of the marginal cdf's. This implies that the copula is unique if the marginals $F_{i}$ are continuous. The converse is also true: given a copula $C:[0,1]^{d} \rightarrow[0,1]$ and margins $F_{i}(x)$ then $C\left(F_{1}\left(x_{1}\right), \ldots, F_{d}\left(x_{d}\right)\right)$ defines a d-dimensional cumulative distribution function.

Visualizing the empirical copula for cryptocurrencies will help us understand the dependence structure of returns. In the following Figures 15 and 16 we will show the three-dimensional empirical copula for our six cryptocurrencies. First, we have Bitcoin, Dash and Litecoin, next we show MaidSafeCoin, Monero and Ripple. We can see that the first of those two copulas shows much a higher correlation than the second one. This is again an indication of the close relationship between Bitcoin and Litecoin and, to a lesser extent, Dash. The second copula exhibits very low correlation and large dispersion, indicating low tail dependence between MaidSafeCoin, Monero and Ripple.

In the following Figure 15, we have plotted the empirical copula for Bitcoin, Dash and Litecoin. 


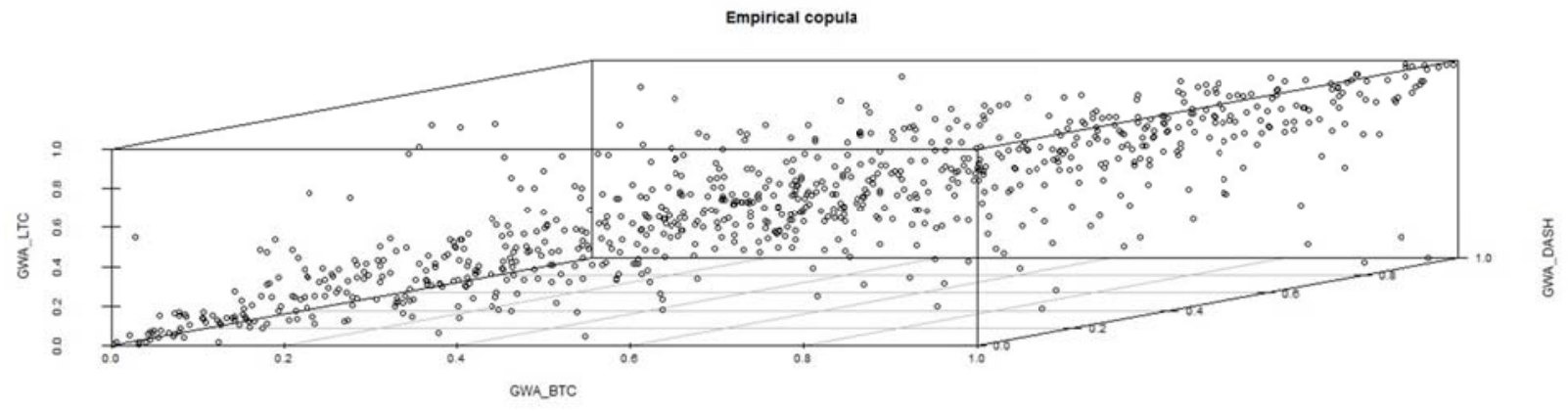

Figure 15. Empirical copula for Bitcoin, Dash, Litecoin

In Figure 16, we have plotted the empirical copula for MaidSafeCoin, Monero and Ripple.

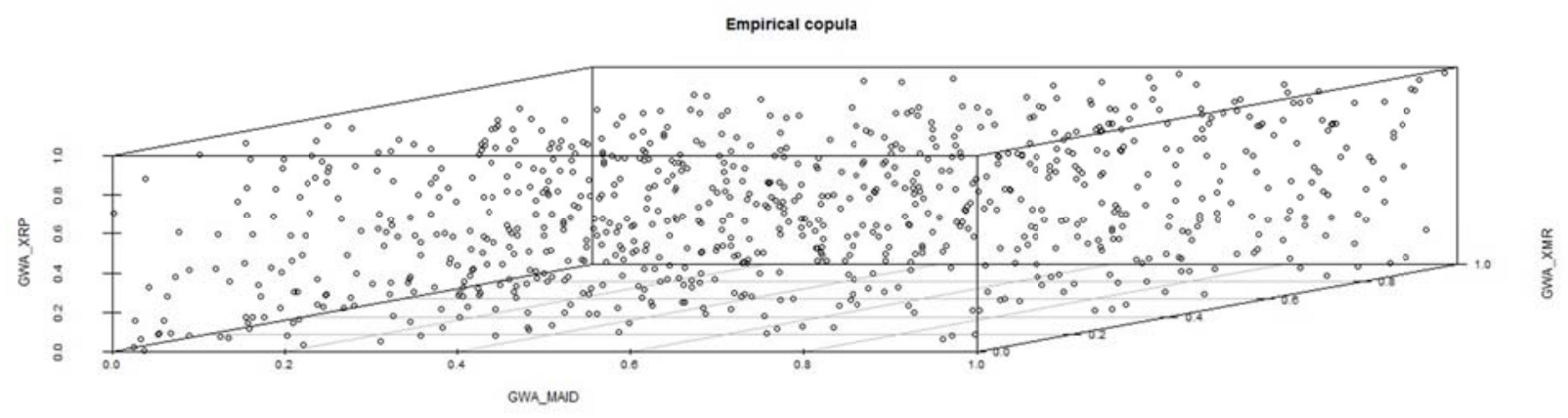

Figure 16. Empirical copula for MaidSafeCoin, Monero and Ripple

Overall, we can reproduce our earlier result, that Bitcoin and Litecoin have higher underlying correlations than the other four cryptocurrencies.

\subsubsection{Gaussian Copula}

We want to fit a parametric copula to the data. For this, we use the Gaussian copula and fit it simultaneously to the six cryptocurrencies under consideration.

Definition 3 (Gaussian copula). The Gaussian copula is a distribution over the unit cube $[0,1]^{d}$. It is constructed from a multivariate normal distribution over $\mathbb{R}^{d}$ by using the probability integral transform. For a given correlation matrix $R \in-[1,1]^{d \times d}$, the Gaussian copula with parameter matrix $R$ can be written as $C_{R}^{\text {Gauss }}(u)=\Phi_{R}\left(\Phi^{-1}\left(u_{1}\right), \ldots, \Phi^{-1}\left(u_{d}\right)\right.$, where $\Phi^{-1}$ is the inverse cumulative distribution function of a standard normal and $\Phi_{R}$ is the joint cumulative distribution function of a multivariate normal distribution with mean vector zero and covariance matrix equal to the correlation matrix $R$. While there is no simple analytical formula for the copula function, $C_{R}^{G a u s s}(u)$, it can be upper or lower bounded, and approximated using numerical integration. The density can be written as 


$$
c_{R}^{\text {Gauss }}(u)=\frac{1}{\sqrt{\operatorname{det}(R)}} \exp \left(-\frac{1}{2}\left(\begin{array}{l}
\Phi^{-1}\left(u_{1}\right) \\
\vdots \\
\Phi^{-1}\left(u_{d}\right)
\end{array}\right)^{T} \cdot\left(R^{-1}-I\right) \cdot\left(\begin{array}{l}
\Phi^{-1}\left(u_{1}\right) \\
\vdots \\
\Phi^{-1}\left(u_{d}\right)
\end{array}\right)\right)
$$

where $\mathbf{I}$ is the identity matrix.

In our case, this leads to a six-dimensional copula for which we record the parameters in Table 6, together with their standard errors in Table 7. The estimation procedure which was used is maximum likelihood and the log-likelihood resulted in 548 .

Table 6. Coefficients of Gaussian copula for cryptocurrencies

\begin{tabular}{|l|l|l|l|l|l|l|}
\hline & Bitcoin & Dash & Litecoin & Maid & Monero & Ripple \\
\hline Bitcoin & 1 & 0.391 & 0.753 & 0.315 & 0.380 & 0.185 \\
\hline Dash & 0.391 & 1 & 0.326 & 0.219 & 0.213 & 0.160 \\
\hline Litecoin & 0.753 & 0.326 & 1 & 0.220 & 0.280 & 0.179 \\
\hline MAID & 0.315 & 0.219 & 0.220 & 1 & 0.201 & 0.120 \\
\hline Monero & 0.380 & 0.213 & 0.280 & 0.201 & 1 & 0.078 \\
\hline Ripple & 0.185 & 0.160 & 0.179 & 0.120 & 0.078 & 1 \\
\hline
\end{tabular}

The standard errors of our Gausian copula:

Table 7. Standard errors of Gaussian copula for cryptocurrencies

\begin{tabular}{|l|l|l|l|l|l|l|}
\hline & Bitcoin & Dash & Litecoin & Maid & Monero & Ripple \\
\hline Bitcoin & 0 & 0.028 & 0.012 & 0.030 & 0.028 & 0.033 \\
\hline Dash & 0.028 & 0 & 0.029 & 0.032 & 0.032 & 0.034 \\
\hline Litecoin & 0.012 & 0.029 & 0 & 0.032 & 0.030 & 0.033 \\
\hline MAID & 0.030 & 0.032 & 0.032 & 0 & 0.033 & 0.034 \\
\hline Monero & 0.028 & 0.032 & 0.030 & 0.033 & 0 & 0.035 \\
\hline Ripple & 0.033 & 0.034 & 0.033 & 0.034 & 0.035 & 0 \\
\hline
\end{tabular}

Also, for visualization purposes, we show the three-dimensional Gaussian copula for Bitcoin, Litecoin and Dash in Figure 17 as well as for MaidSafeCoin, Ripple and Moneroin Figure 18. 


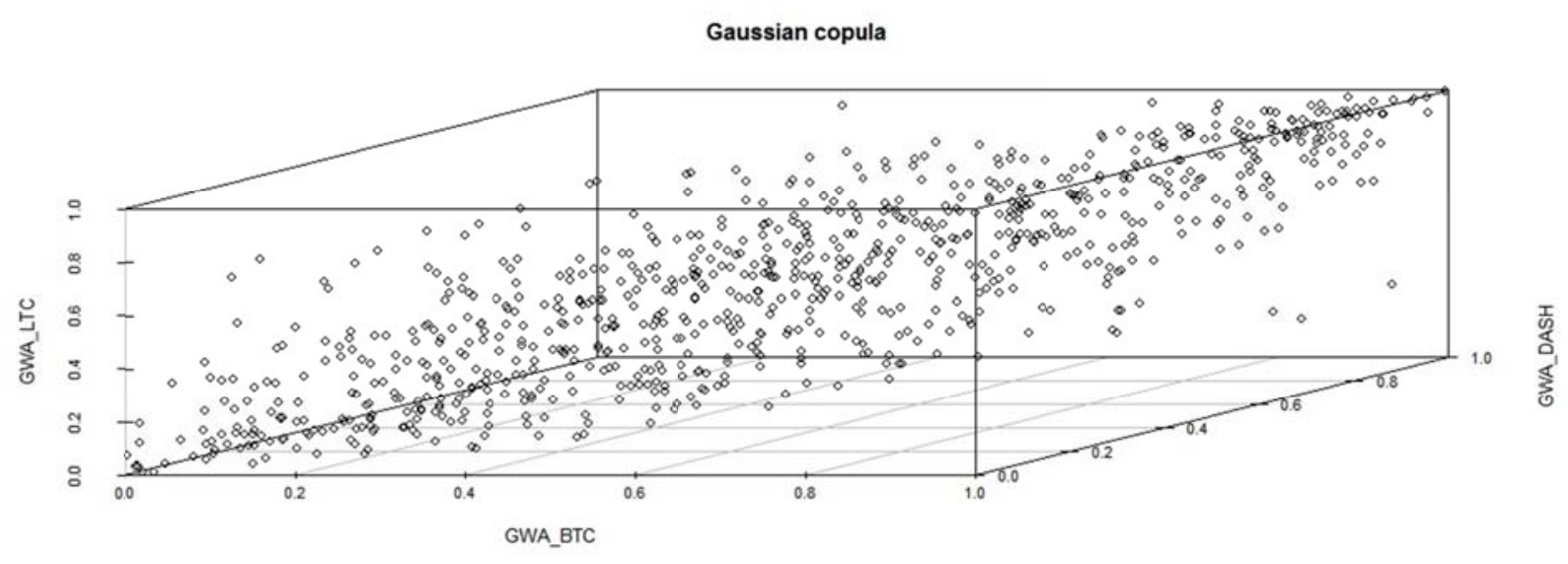

Figure 17. Gaussian copula for Bitcoin, Dash, Litecoin

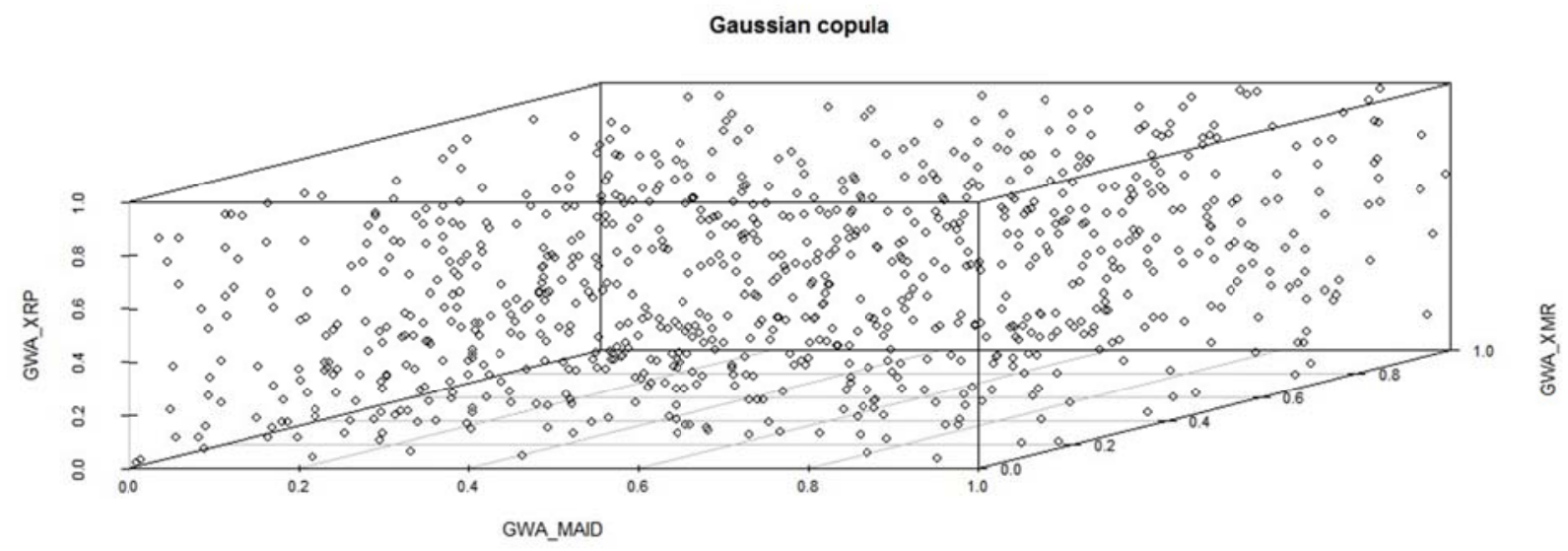

Figure 18. Gaussian copula for MaidSafeCoin, Monero and Ripple

\subsection{Tail Dependence Coefficient}

Apart from the correlation of the cryptocurrency returns, looking at the behaviour of them in extreme events is of utmost importance. For this purpose we are considering the tail dependence of the cryptocurrency returns. First, we compute the matrix of tail-dependence coefficients, using the nonparametric estimator of (Schmid \& Schmidt, 2007) (see also (Jaworski, Durante, \& Hardle, 2010), computed for all pairs of returns.

The results for the lower tail dependence can be seen in Table 8: 
Table 8. Lower tail dependence of cryptocurrencies

\begin{tabular}{|l|l|l|l|l|l|l|}
\hline & Bitcoin & Dash & Litecoin & Maid & Monero & Ripple \\
\hline Bitcoin & 1 & 0.300 & 0.650 & 0.272 & 0.368 & 0.151 \\
\hline Dash & 0.300 & 1 & 0.270 & 0.116 & 0.164 & 0.024 \\
\hline Litecoin & 0.650 & 0.270 & 1 & 0.187 & 0.318 & 0.156 \\
\hline MAID & 0.272 & 0.116 & 0.187 & 1 & 0.177 & 0.079 \\
\hline Monero & 0.368 & 0.164 & 0.318 & 0.177 & 1 & 0.104 \\
\hline Ripple & 0.151 & 0.024 & 0.156 & 0.079 & 0.104 & 1 \\
\hline
\end{tabular}

The cut-off parameter $p$ in $[0,1]$ below which the estimation takes place is chosen as $p=\min (100 /$ nobs, 0.1$)$, were nobs stands for the number of observations. Bitcoin and Litecoin have the highest tail dependence, whereas Ripple has the lowest tail dependence with all other cryptocurrencies.

We are also computing the lower tail dependence coefficient by fitting pairwise $t$ copulas to the data and returning the implied tail-dependence coefficient in Table 9. Details of it can be found in (Jaworski, Durante, \& Hardle, 2010) and (Schmid \& Schmidt, 2007).

Table 9. Lower tail dependence of cryptocurrencies, fitting a t-copula

\begin{tabular}{|l|l|l|l|l|l|l|}
\hline & Bitcoin & Dash & Litecoin & Maid & Monero & Ripple \\
\hline Bitcoin & 1 & 0.140 & 0.479 & 0.102 & 0.053 & 0.046 \\
\hline Dash & 0.140 & 1 & 0.082 & 0.048 & 0.100 & 0.001 \\
\hline Litecoin & 0.479 & 0.082 & 1 & 0.033 & 0.020 & 0.048 \\
\hline MAID & 0.102 & 0.048 & 0.033 & 1 & 0.107 & 0.000 \\
\hline Monero & 0.053 & 0.100 & 0.020 & 0.107 & 1 & 0.005 \\
\hline Ripple & 0.046 & 0.001 & 0.048 & 0.000 & 0.005 & 1 \\
\hline
\end{tabular}

Using a t-copula, there is only very low tail dependency between all cryptocurrencies, except for Bitcoin and Litecoin.

\subsection{Value-at-risk and Expected Shortfall}

We want to characterize the losses that can occur in extreme events when investing in cryptocurrencies. The two most important tail risk measures are value-att-risk and expected shortfall.

Definition 4 (at Risk). Mathematically, if $X$ is a random variable (e.g., the price of a portfolio), then $\operatorname{VaR}_{\alpha}(X)$ is the negative of the $\alpha$-quantile, i.e.,

$$
\operatorname{VaR}_{\alpha}(X)=\inf \{x \in \mathbb{R} \mid P(X<-x) \leq 1-\alpha\} .
$$


Definition 5 (shortfall). Expected shortfall estimates the potential size of the loss exceeding $V a R_{\alpha}$. For a given random variable $X$, the expected shortfall is defined as the expected size of a loss that exceeds $\operatorname{VaR}_{\alpha}$ :

$$
E\left(S_{\alpha}\right)=E\left(X \mid X>\operatorname{VaR}_{\alpha}\right)
$$

(Artzner, Delbaen, Eber, \& Heath, 1999) argue that expected shortfall, as opposed to value-at-risk, is a coherent risk measure.

In Table 10, we record the value-at-risk at the 95\% level for Bitcoin and the other five cryptocurrencies under consideration, using two methods: First, the historical value-at-risk, which corresponds to taking the $95 \%$ quantile of the negative exchange rates. Second, the Gaussian value-at-risk, which is computed by first fitting a normal distribution to the data, obtaining the mean $\mu$ and standard deviation $\sigma$, and then computing analytically the expected shortfall, using the formula

$$
\operatorname{VaR}_{0.95}^{\mathrm{Gauss}}=\mu+\sigma \cdot q_{\mathrm{norm}}(0.95)
$$

where $q_{\text {norm }}(0.95)$ denotes the $95 \%$ quantile of the standard normal distribution.

Table 10. Value-at-risk for cryptocurrencies, left tail

\begin{tabular}{|l|l|l|}
\hline Exchange rate & Historical VaR & Gaussian VaR \\
\hline Bitcoin/USD & 0.048 & 0.054 \\
\hline Dash/USD & 0.106 & 0.101 \\
\hline Litecoin/USD & 0.068 & 0.083 \\
\hline Maid/USD & 0.125 & 0.123 \\
\hline Monero/USD & 0.125 & 0.132 \\
\hline Ripple/USD & 0.101 & 0.103 \\
\hline
\end{tabular}

We see that the value-at-risk for MaidSafeCoin and Monero are the highest of all, being almost three times as large as the one for Bitcoin. You can expect to lose more than $12 \%$ in one day, about once every 20 days, when you are invested in Monero. For Bitcoin, this would translate to losing more than $4.8 \%$ once every 20 days. Comparing historical VaR and Gaussian VaR, we can observe that those two numbers usually differ. Gaussian VaR is much higher than the historical VaR. This shows the deviation of the cryptocurrency exchange rates from the Gaussian distribution and the higher risks that they bear.

In Table 11 below, we record the expected shortfall at the $95 \%$ level for our cryptocurrencies, using two methods: First, the historical expected shortfall, which corresponds to computing the expected shortfall from the historical sample.

Second, the Gaussian expected shortfall, which is computed by first fitting a normal distribution to the data, obtaining the $\mu$ and standard deviation $\sigma$, and then computing analytically the expected shortfall, using the formula 


$$
E S_{0.95}=\mu+\sigma \cdot E S_{\text {norm }}(0.95)
$$

where $E S_{\text {norm }}(0.95)$ denotes the $95 \%$ expected shortfall of the standard normal distribution.

Table 11. Expected shortfall for cryptocurrencies, left tail

\begin{tabular}{|l|l|l|}
\hline Exchange rate & Historical ES & Gaussian ES \\
\hline Bitcoin/USD & 0.077 & 0.067 \\
\hline Dash/USD & 0.168 & 0.127 \\
\hline Litecoin/USD & 0.135 & 0.104 \\
\hline Maid/USD & 0.194 & 0.153 \\
\hline Monero/USD & 0.213 & 0.164 \\
\hline Ripple/USD & 0.180 & 0.128 \\
\hline
\end{tabular}

We see that the expected shortfall is highest for Monero and about three times larger than for Bitcoin. Provided you find yourself on one of those 1-in-20 days where you can expect to lose more than $12 \%$ in Monero, you will actually end up losing about $21.3 \%$ on that day, a truly risky investment. Comparing historical ES and Gaussian ES, we can observe that the Gaussian ES is lower than the historical ES, indicating a higher risk in cryptocurrencies than one would expect from a normal distribution. For completeness, we also record the value-at-risk and the expected shortfall for the right tail (with the sign reversed), indicating the risk of being long USD and short the cryptocurrency. Those values are in Table 12 and 13 respectively.

Table 12. Expected shortfall for cryptocurrencies, right tail

\begin{tabular}{|l|l|l|}
\hline Exchange rate & Historical VaR & Gaussian VaR \\
\hline Bitcoin/USD & 0.047 & 0.053 \\
\hline Dash/USD & 0.083 & 0.099 \\
\hline Litecoin/USD & 0.070 & 0.084 \\
\hline Maid/USD & 0.102 & 0.113 \\
\hline Monero/USD & 0.103 & 0.125 \\
\hline Ripple/USD & 0.079 & 0.098 \\
\hline
\end{tabular}

Expected shortfall for the right tail, at the 95\% level: 
Table 13. Expected shortfall for cryptocurrencies, right tail

\begin{tabular}{|l|l|l|}
\hline Exchange rate & Historical ES & Gaussian ES \\
\hline Bitcoin/USD & 0.082 & 0.066 \\
\hline Dash/USD & 0.129 & 0.124 \\
\hline Litecoin/USD & 0.116 & 0.105 \\
\hline Maid/USD & 0.133 & 0.144 \\
\hline Monero/USD & 0.144 & 0.157 \\
\hline Ripple/USD & 0.128 & 0.123 \\
\hline
\end{tabular}

Bitcoin is risky and other cryptocurrencies are even riskier.

\subsection{Extremal Index}

The extremal index $\theta$ is a useful indicator of how much clustering of exceedances of a threshold occurs in the limit of the distribution. For independent data, $\theta=1$, (though the converse does not hold) and if $\theta<1$, then there is some dependency (clustering) in the limit. There are many possible estimators of the extremal index. The ones used here are runs declustering (e.g., Coles \& Coles, 2001; section 5.3.2) and the intervals estimator described in (Ferro \& Segers, 2003). It is unbiased in the mean and can be used to estimate the number of clusters. In the following table 14 we show the extremal index for the left tail of the cryptocurrency returns. This is computed for the $90 \%$ quantile, using a run length of four for the runs method.

Extremal index for the left tail:

Table 14. Extremal index for negative returns $90 \%$ quantile

\begin{tabular}{|l|l|l|l|l|l|l|}
\hline Exchange rate & \multicolumn{4}{|l|}{ Runs declustering } & \multicolumn{2}{l|}{ Intervals estimator } \\
\hline & $\theta$ & \#clusters & run length & $\theta$ & \#clusters & run length \\
\hline Bitcoin/USD & 0.578 & 48 & 4 & 0.728 & 54 & 3 \\
\hline Dash/USD & 0.506 & 42 & 4 & 0.539 & 42 & 4 \\
\hline Litecoin/USD & 0.542 & 45 & 4 & 0.787 & 58 & 2 \\
\hline Maid/USD & 0.639 & 53 & 4 & 0.946 & 73 & 1 \\
\hline Monero/USD & 0.602 & 50 & 4 & 0.854 & 64 & 2 \\
\hline Ripple/USD & 0.554 & 46 & 4 & 0.725 & 60 & 2 \\
\hline
\end{tabular}

Table 15 shows the extremal index for the right tail: 


\section{Macrothink}

Table 15. Extremal index for positive returns $90 \%$ quantile

\begin{tabular}{|l|l|l|l|l|l|l|}
\hline \multirow{2}{*}{$\begin{array}{l}\text { Exchange } \\
\text { rate }\end{array}$} & \multicolumn{4}{|l|}{ Runs declustering } & \multicolumn{3}{l|}{ Intervals estimator } \\
\cline { 2 - 7 } & $\theta$ & \#clusters & run length & $\theta$ & \#clusters & run length \\
\hline Bitcoin/USD & 0.482 & 40 & 4 & 0.564 & 46 & 3 \\
\hline Dash/USD & 0.554 & 46 & 4 & 0.589 & 46 & 4 \\
\hline Litecoin/USD & 0.530 & 44 & 4 & 0.614 & 51 & 3 \\
\hline Maid/USD & 0.614 & 51 & 4 & 0.627 & 51 & 4 \\
\hline Monero/USD & 0.590 & 49 & 4 & 0.771 & 58 & 3 \\
\hline Ripple/USD & 0.494 & 41 & 4 & 0.695 & 56 & 2 \\
\hline
\end{tabular}

As far as clustering of extreme events is concerned, there is clear evidence for that to happen in cryptocurrencies, in particular in the right tail. Comparing the extremal indices for different currencies, Dash has the most clustering in the left tail (i.e., when being long Dash, you would expect large losses to be clustered). In the right tail, Bitcoin shows the largest clustering effect.

\section{Extreme Value Distributions}

Extreme value distributions are distributions characterizing the tails of a distribution. Since we are interested in the risk characteristics of cryptocurrencies, we are focusing on extreme events, in particular very large negative returns. In extreme value theory, two distributions play an important role: the generalized Pareto distribution and the generalized extreme value distribution.

\subsection{Cryptocurrencies and the Generalized Pareto Distribution}

The generalized Pareto distribution (GPD) is a family of continuous probability distributions. It is used to model the tails of a distribution and specified by three parameters: location $\mu$, scale $\beta$, and shape $\xi$.

Definition 6 (Generalized Pareto distribution). The cumulative probability distribution function of a generalized Pareto distribution is given by

$$
F_{(\xi, \mu, \beta)}=\left\{\begin{array}{l}
1-\left(1+\frac{\xi(x-\mu)}{\beta}\right)^{-\frac{1}{\xi}} \text { for } \xi \neq 0 \\
1-\exp \left(-\frac{x-\mu}{\beta}\right) \text { for } \xi=0
\end{array}\right.
$$

for $x \geq \mu$ when $\xi \geq 0$, and $\mu \leq x \leq \mu-\beta / \xi$ when $\xi<0$, where $\mu \in \mathbb{R}, \beta>0$ and $\xi \in \mathbb{R}$.

The Pickands-Balkema-de Haan theorem is often called the second theorem in extreme value theory. It gives the asymptotic tail distribution of a random variable $\mathrm{X}$, when the true distribution $\mathrm{F}$ of $\mathrm{X}$ is unknown. Unlike for the first theorem (the Fisher-Tippett-Gnedenko 
theorem) in extreme value theory, the interest here is in the values above a threshold.

The Pickands-Balkema-de Haan theorem is often called the second theorem in extreme value theory. It gives the asymptotic tail distribution of a random variable $\mathrm{X}$, when the true distribution $\mathrm{F}$ of $\mathrm{X}$ is unknown. Unlike for the first theorem (the Fisher-Tippett-Gnedenko theorem) in extreme value theory, the interest here is in the values above a threshold.

Theorem 2 (Pickands-Balkema-de Haan) Let $\left(X_{1}, \ldots, X_{n}\right)$ be a sequence of independent and identically-distributed random variables, and let $F_{u}$ be their conditional excess distribution function. In (Balkema \& de Haan, 1974) and (Pickands, 1975), Pickands, Balkema and de Haan show that for a large class of underlying distribution functions $F$, and large $u, F_{u}$ is well approximated by the generalized Pareto distribution. That is:

where

$$
F_{u}(y) \rightarrow G_{k, \sigma}(y), \quad \text { as } u \rightarrow \infty
$$

$$
G_{k, \sigma}(y)=1-\left(1+\frac{k y}{\sigma}\right)^{-\frac{1}{k}}
$$

if $k \neq 0$ and

$$
G_{k, \sigma}(y)=1-\exp \left(-\frac{y}{\sigma}\right)
$$

if $k=0$. Here $\sigma>0$, and $y \geq 0$ when $k \geq 0$ and $0 \leq y \leq-\sigma / k$ when $k<0$. Since a special case of the generalized Pareto distribution is a power-law, the Pickands-Balkema-de Haan theorem is sometimes used to justify the use of a power-law for modeling extreme events. We are fitting a GPD distribution to the 150 largest daily negative returns, i.e., the left tail of the distribution of returns. The parameters, together with their standard errors, the threshold value which is implied by those 150 returns as well as the negative log-likelihood is given in table 16. The estimation procedure is using the maximum likelihood method and is based on (Hosking \& Wallis, 1987).

Table 16. Maximum likelihood fit of generalized Pareto distribution

\begin{tabular}{|l|l|l|l|l|l|l|}
\hline Exchange rate & \multicolumn{1}{|c|}{$\xi$} & \multicolumn{1}{|c|}{$\xi$} & \multicolumn{1}{c|}{$\xi$ s.e. } & \multicolumn{1}{c|}{$\beta$ s.e. } & \multicolumn{1}{c|}{ threshold } & nllh \\
\hline Bitcoin/ USD & 0.272 & 0.019 & 0.115 & 0.003 & 0.018 & -406 \\
\hline Dash/ USD & 0.111 & 0.035 & 0.095 & 0.004 & 0.036 & -338 \\
\hline Litecoin/ USD & 0.233 & 0.029 & 0.109 & 0.004 & 0.022 & -345 \\
\hline Maid/ USD & -0.043 & 0.040 & 0.061 & 0.004 & 0.050 & -339 \\
\hline Monero/ USD & -0.042 & 0.044 & 0.080 & 0.005 & 0.050 & -325 \\
\hline Ripple/ USD & 0.264 & 0.029 & 0.113 & 0.004 & 0.031 & -341 \\
\hline
\end{tabular}

The cryptocurrency Bitcoin has the highest shape parameter $\xi$, with a vallue of 0.272 and a standard error of 0.115 . For illustrative purposes, we show the density of a GPD distribution with the shape parameter $\xi=0.272$ and the scale parameter $\beta=0.019$ (Bitcoin/ USD, Figure 19) versus the parameters $\xi=-0.042$ and $\beta=0.044$ (Monero/ USD, Figure 20) 


\section{Macrothink}

in the following chart so that the difference between the Bitcoin/ USD exchange rate and Monero/ USD becomes obvious from a graphical point of view. We also see that Bitcoin, Litecoin and Ripple have similar parameters. Furthermore, Monero and MaidSafeCoin are similar. Dash is in the middle of those two groups.

The density of the GPD distribution fitted to the Bitcoin/ USD exchange rate:

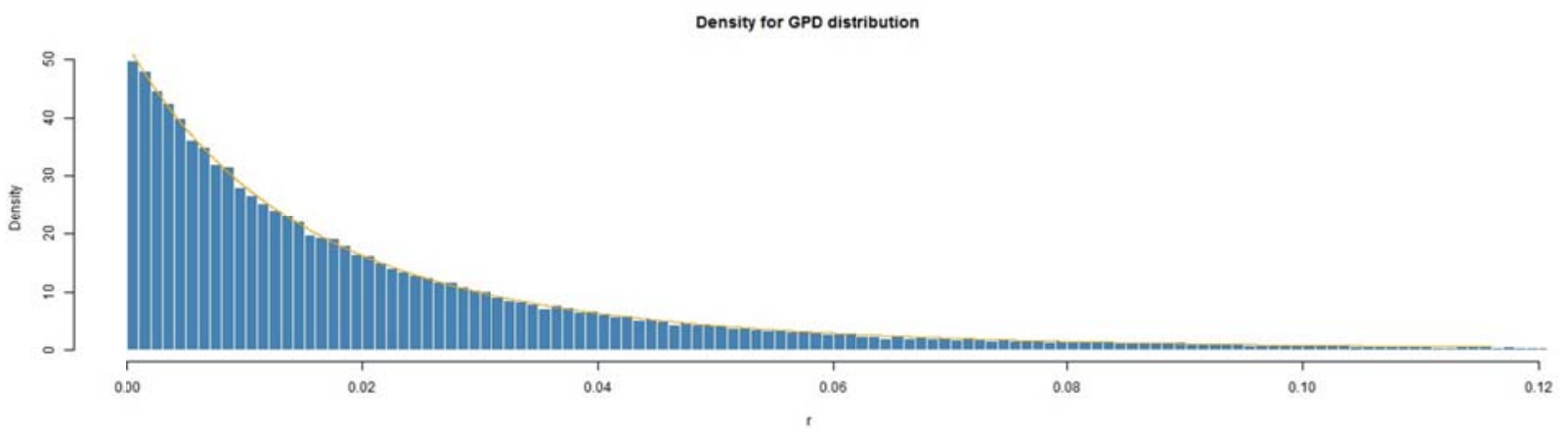

Figure 19. Density of a GPD distribution with parameters $\xi=0.272$ and $\beta=$ 0.019(Bitcoin)

The density of the GPD distribution fitted to the Monero/ USD exchange rate:

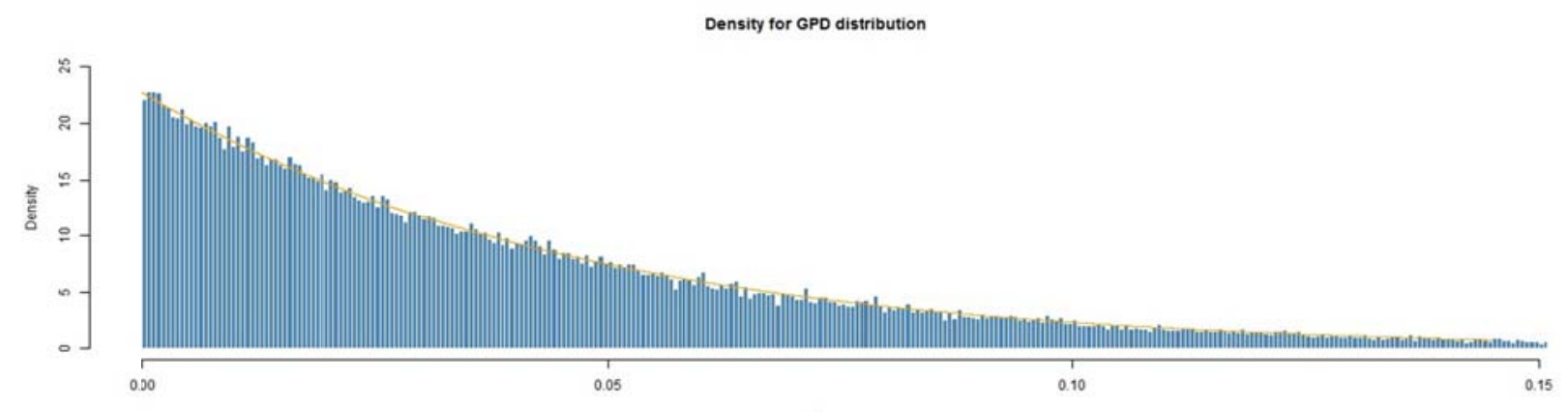

Figure 20. Density of a GPD distribution with parameters $\xi=-0.042$ and $\beta=$ 0.044(Monero)

By comparing both Figures 19 and 20, and looking at the scales of the x-axis and the y-axis, we see that the GPD distribution for the Bitcoin/ USD exchange rate has much larger $\mathrm{x}$-values than the one for Monero/ USD. This is indicative for the Bitcoin exchange rate being much riskier than the Monero/USD exchange rate during extreme events.

For sake of completeness, we also show the GPD distribution for the other four 
cryptocurrencies, using the fitted parameters from Table 16:

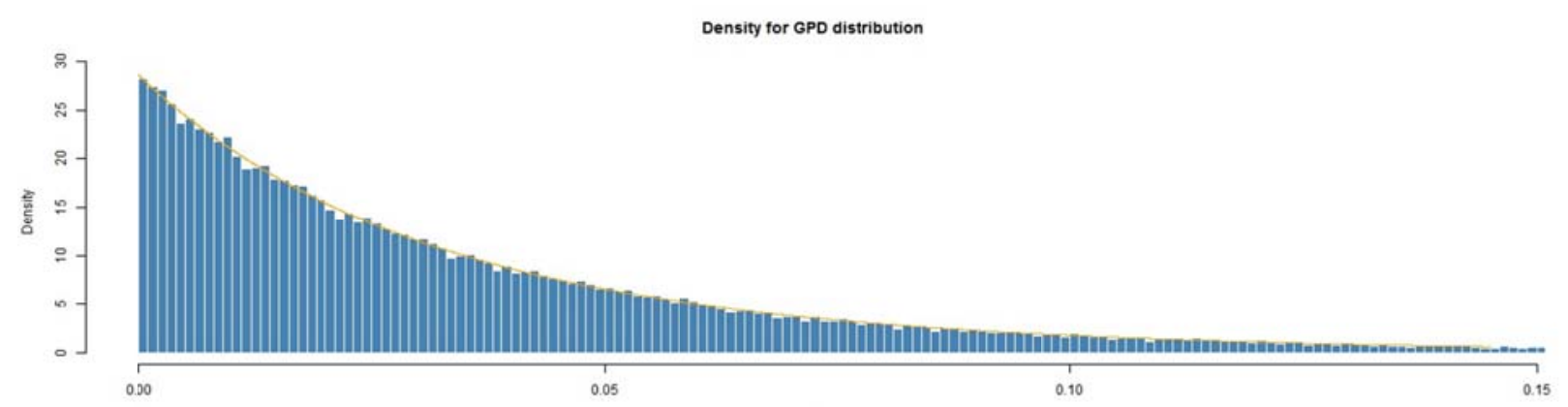

Figure 21. Density of a GPD distribution with parameters $\xi=0.111$ and $\beta=0.035$ (Dash)

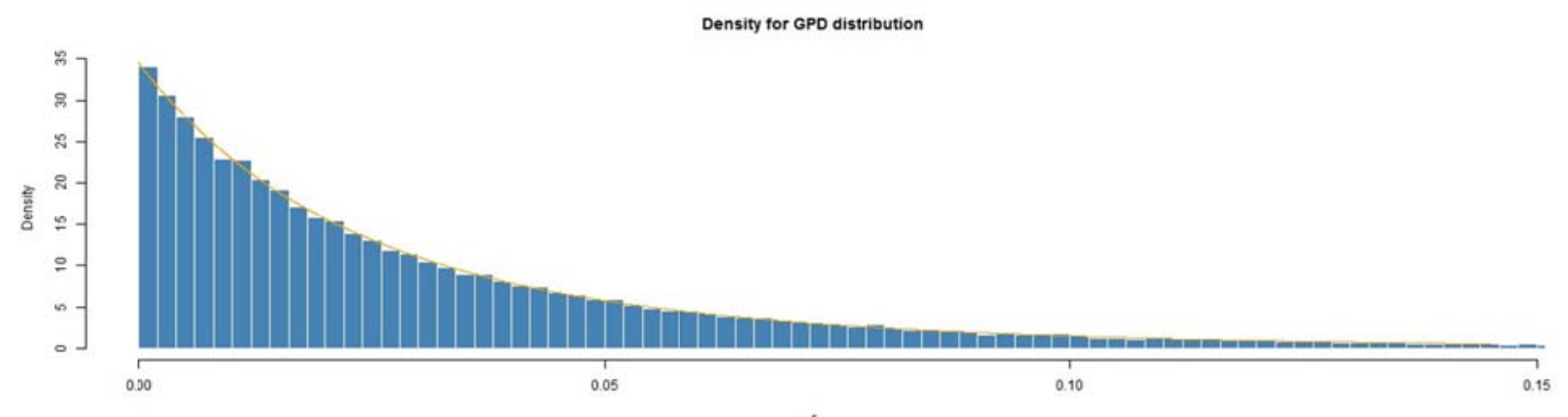

Figure 22. Density of a GPD distribution with parameters $\xi=0.233$ and $\beta=$ 0.029(Litecoin)

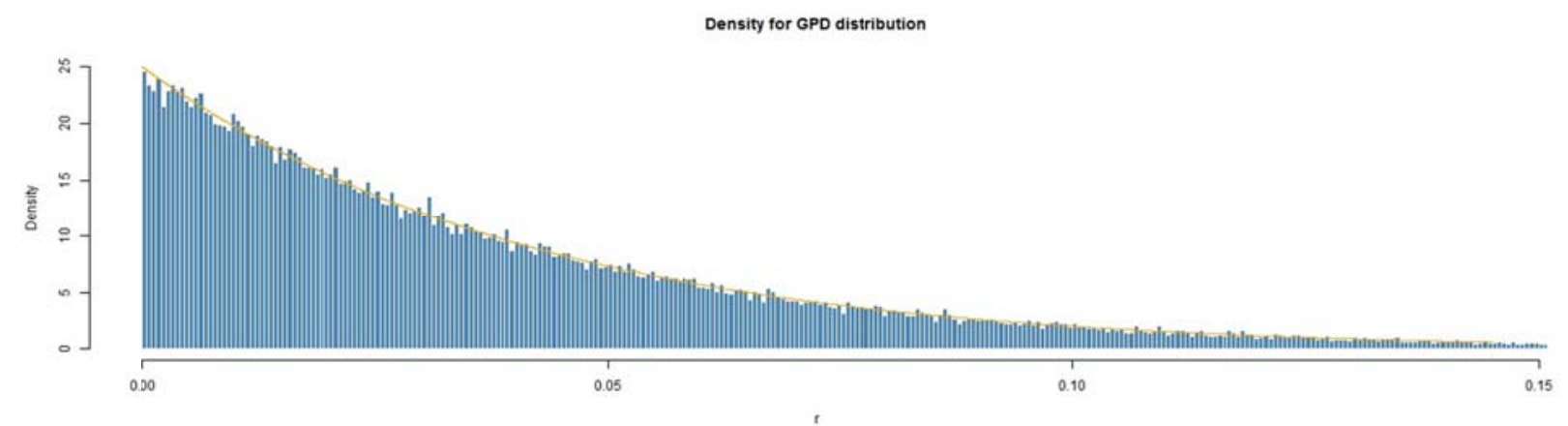

Figure 23. Density of a GPD distribution with parameters $\xi=-0.043$ and $\beta=0.04$ (Maid) 


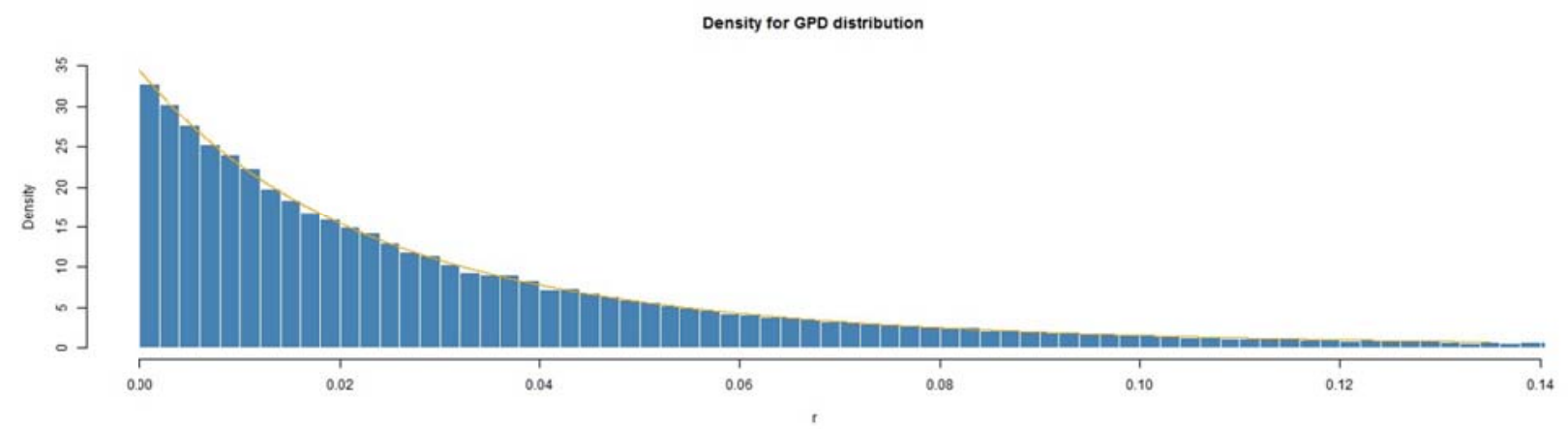

Figure 24. Density of a GPD distribution with parameters $\xi=0.264$ and $\beta=0.029$ (Ripple)

We also show the plots of the cumulative excess distribution func tions $F_{u}(x-u)$ for the $u$ that corresponds to 150 extremes of the cryptocurrency returns. The corresponding threshold value $u$ is given in table 16 .

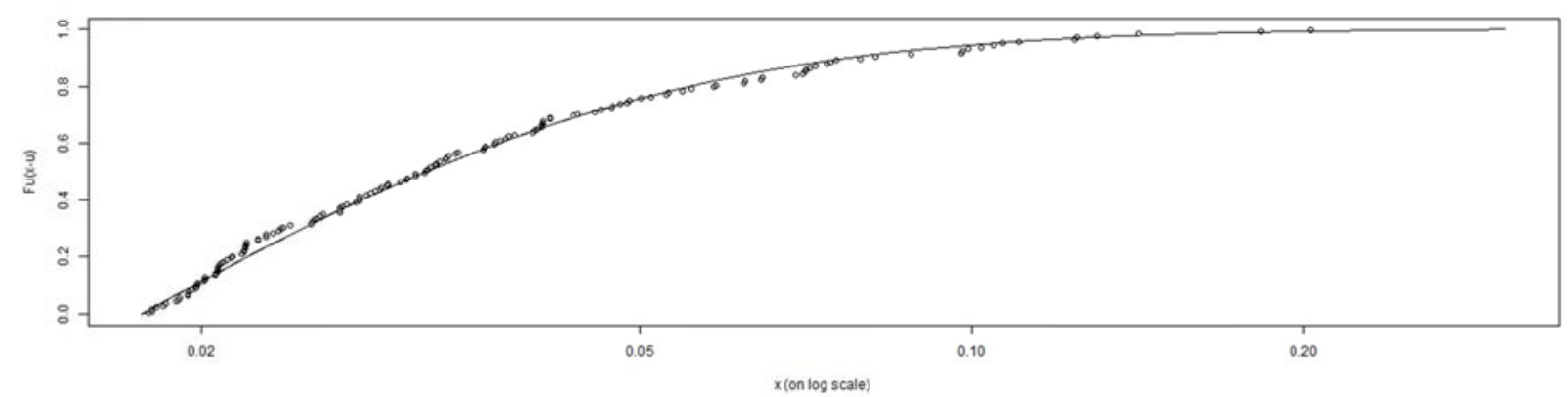

Figure 25. Excess distribution function for Bitcoin

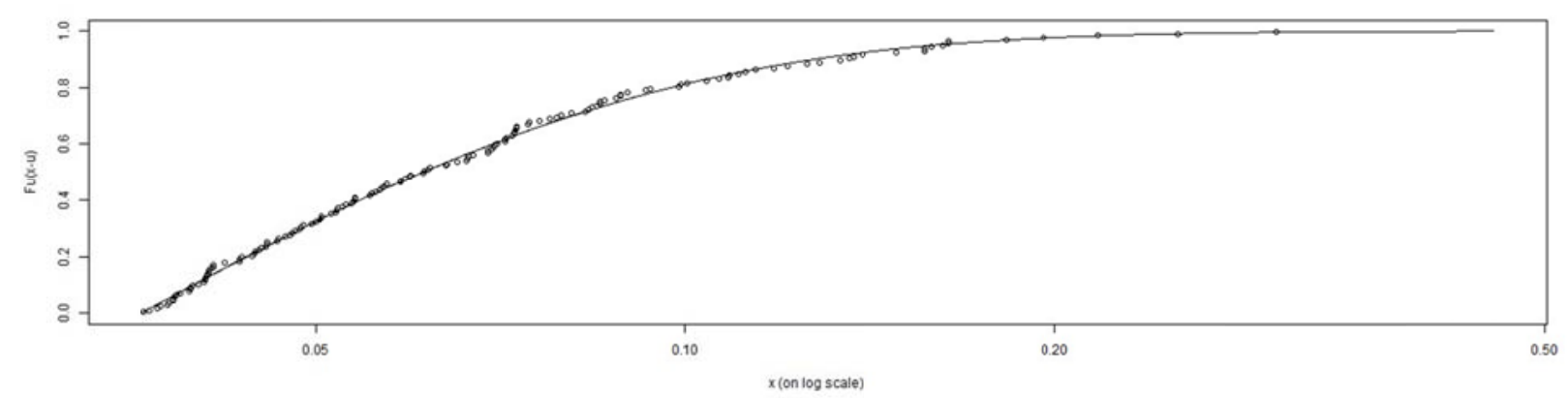

Figure 26. Excess distribution function for Dash 


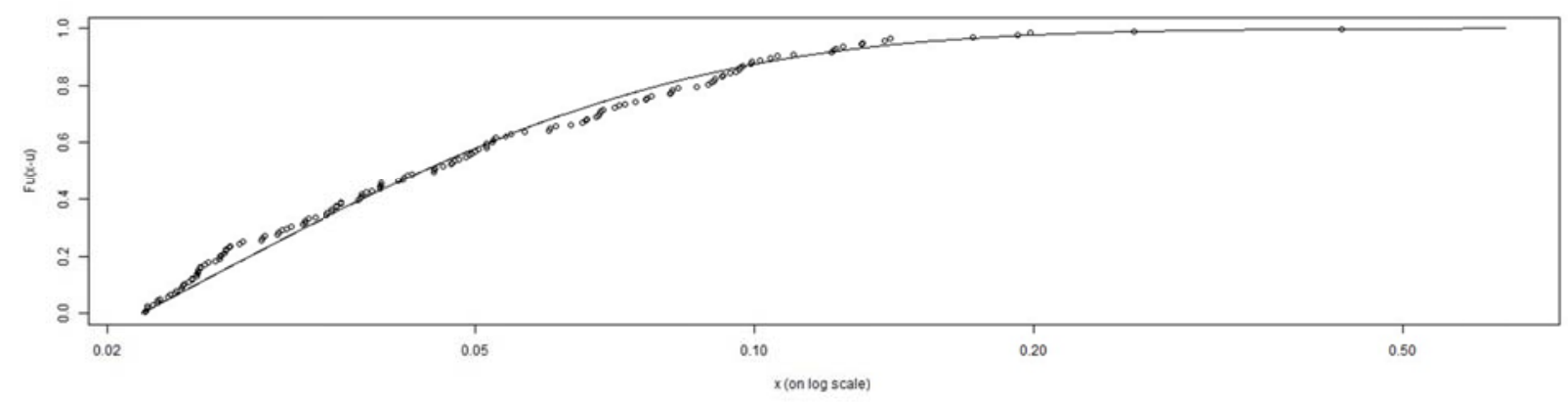

Figure 27. Excess distribution function for Litecoin

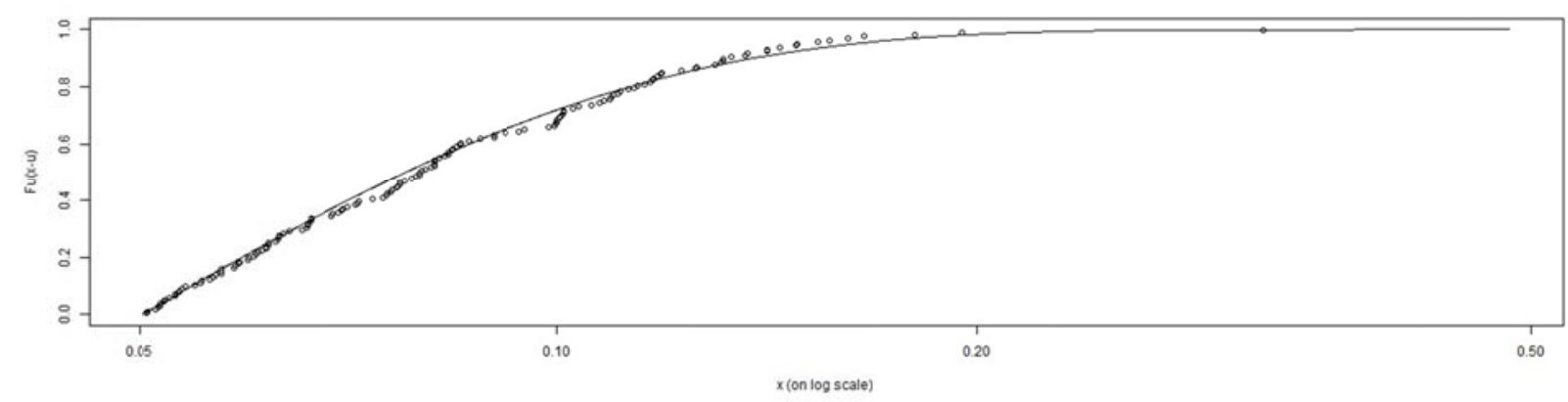

Figure 28. Excess distribution function for Maid

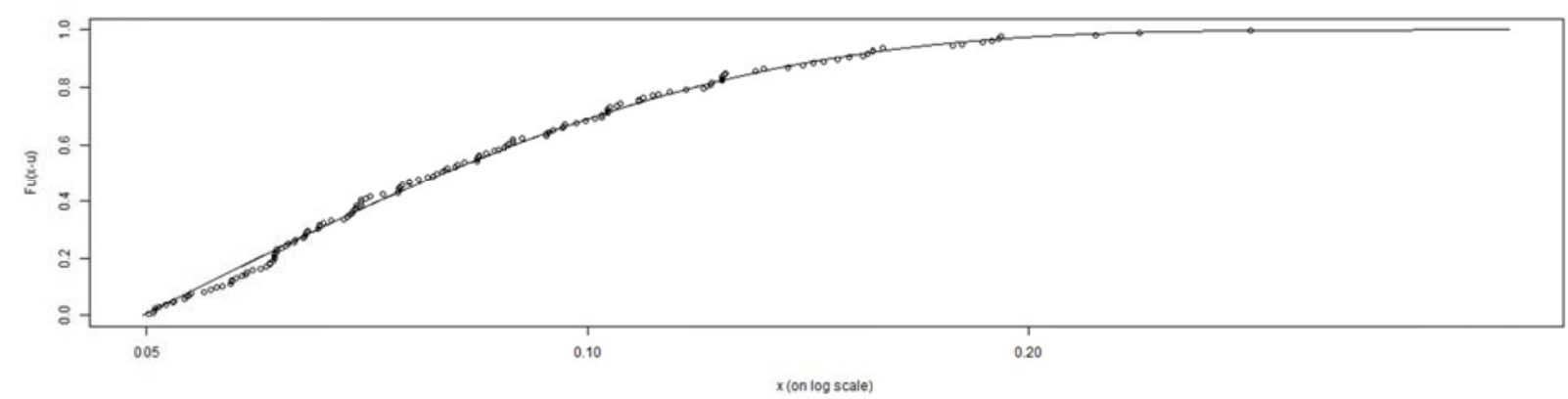

Figure 29. Excess distribution function for Monero

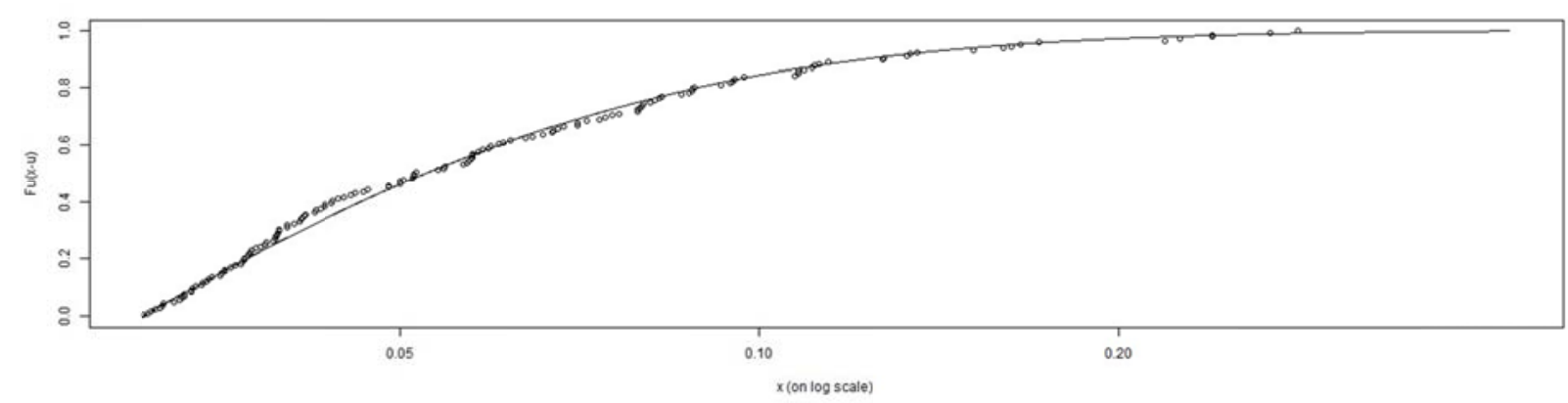

Figure 30. Excess distribution function for Ripple 


\section{IIMacrothink}

Using the mean excess plot, one can visually decide on the appropriate choice of a threshold. In Figure 31 we have plotted the mean excess plot of the negative of the Bitcoin returns, which, for a given threshold $u$, plots the mean value of all returns exceeding $u$. We are also showing a vertical line at $u=0$ and the mean excess line given by fitting a GPD distribution to the values exceeding $u$, obtaining the scale and shape parameters $\beta$ and $\xi$ with the values $\beta=0.016$ and $\xi=0.239$. Once those parameters are obtained, we draw a straight line given by $(\beta+\xi \cdot u) /(1-\xi)$ (the blue straight line in Figure 31$)$.

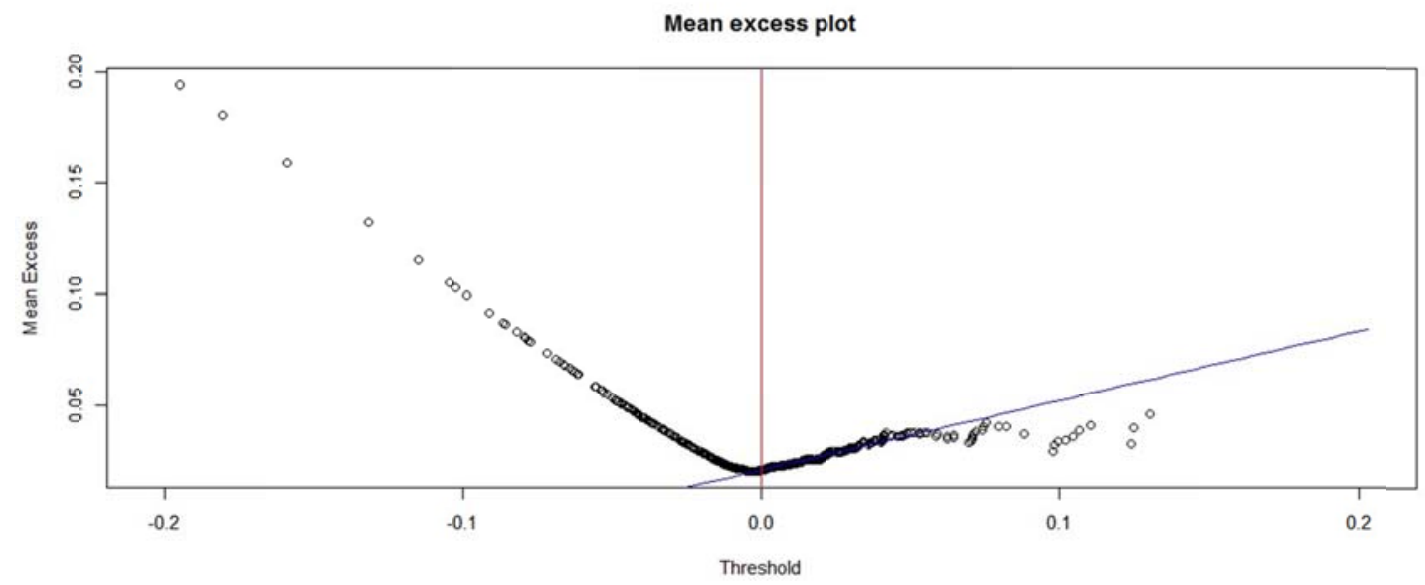

Figure 31. Mean excess plot for the Bitcoin/ USD exchange rate with a vertical line at the threshold $u=0$

An upward trend in the plot shows heavy-tailed behaviour. In particular, a straight line with positive gradient above some threshold is a sign of Pareto behaviour in the tail. A downward trend shows thin-tailed behaviour whereas a line with zero gradient shows an exponential tail.

For comparison, we also show the mean excess plot for the exchange rate between Monero and USD in Figure 32. Here, a threshold of $u=0$ is chosen for the mean excess line. The corresponding parameters are $\beta=0.051$ and $\xi=-0.087$. 


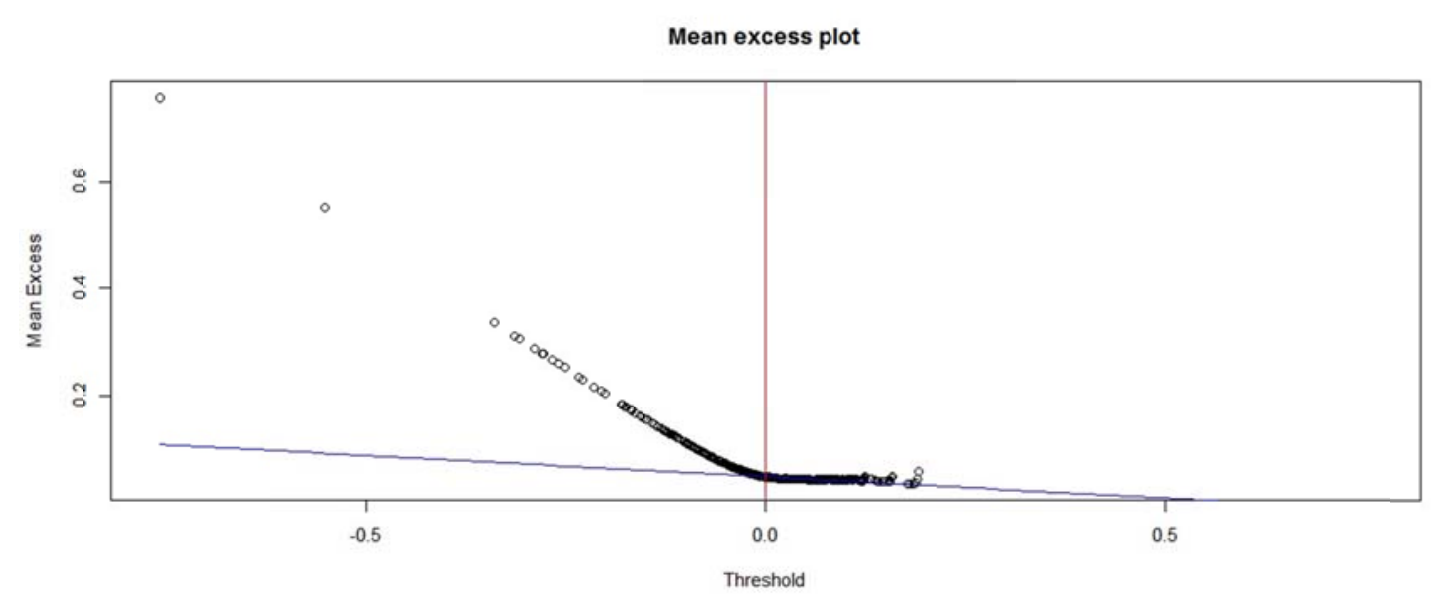

Figure 32. Mean excess plot for the Monero/ USD exchange rate with a vertical line at the threshold $u=0$

In Figures 33, 34, 35, and 36 we are looking at the mean excess plots for the other four cryptocurrencies.

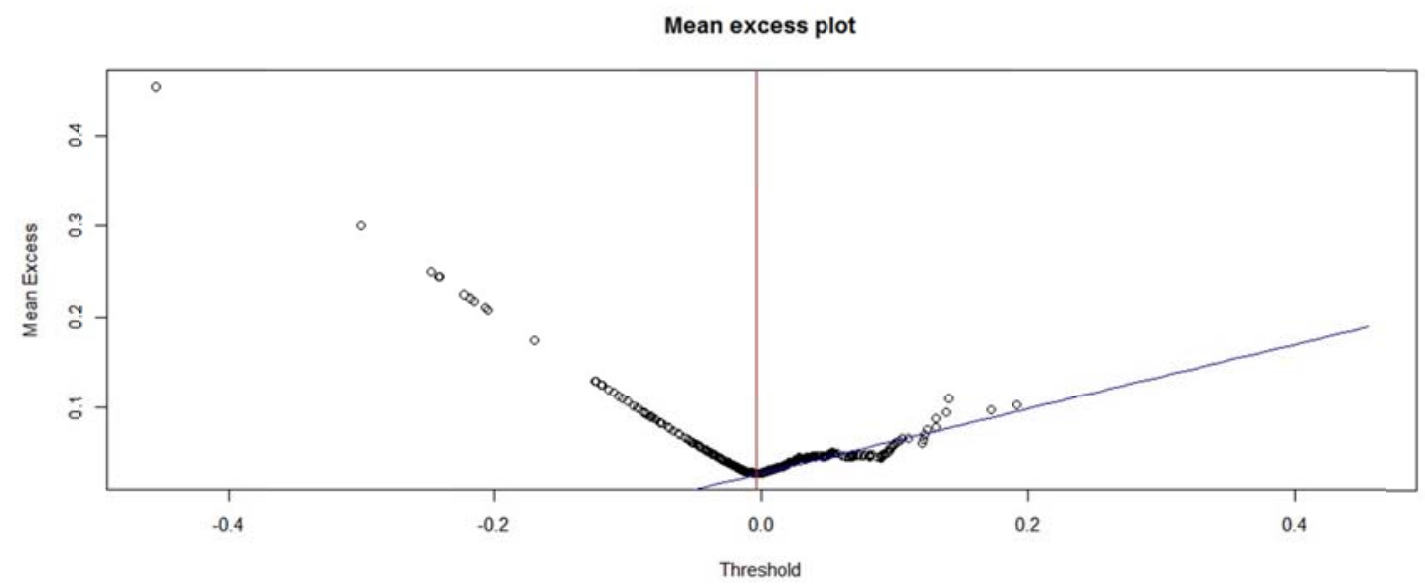

Figure 33. Mean excess plot for the Litecoin/ USD exchange rate with a vertical line at the threshold $u=-0.004$ 


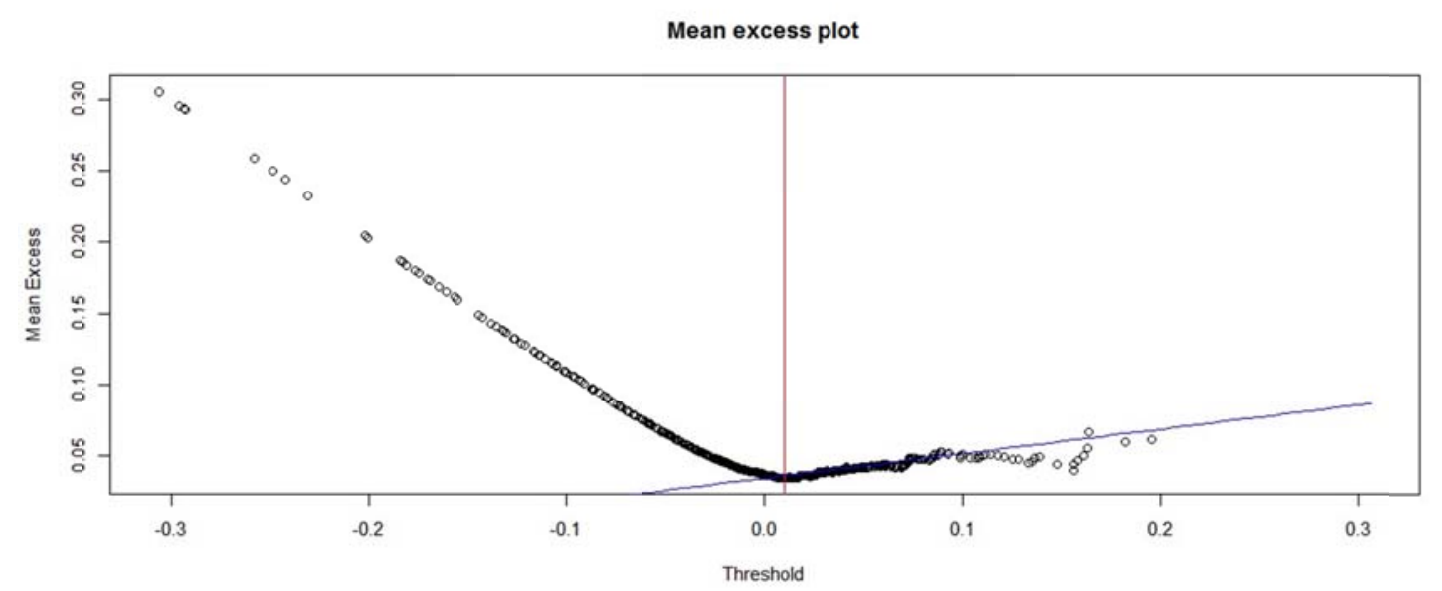

Figure 34. Mean excess plot for the Dash/ USD exchange rate with a vertical line at the threshold $u=0.01$

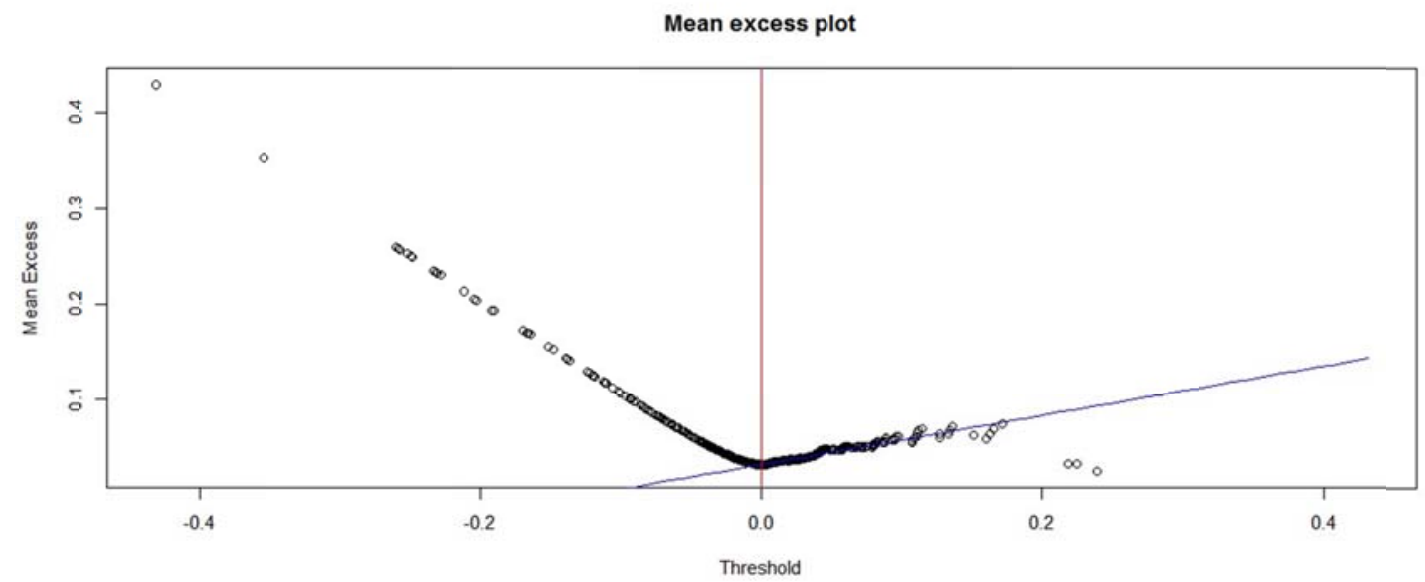

Figure 35. Mean excess plot for the Ripple/ USD exchange rate with a vertical line at the threshold $u=0$ 


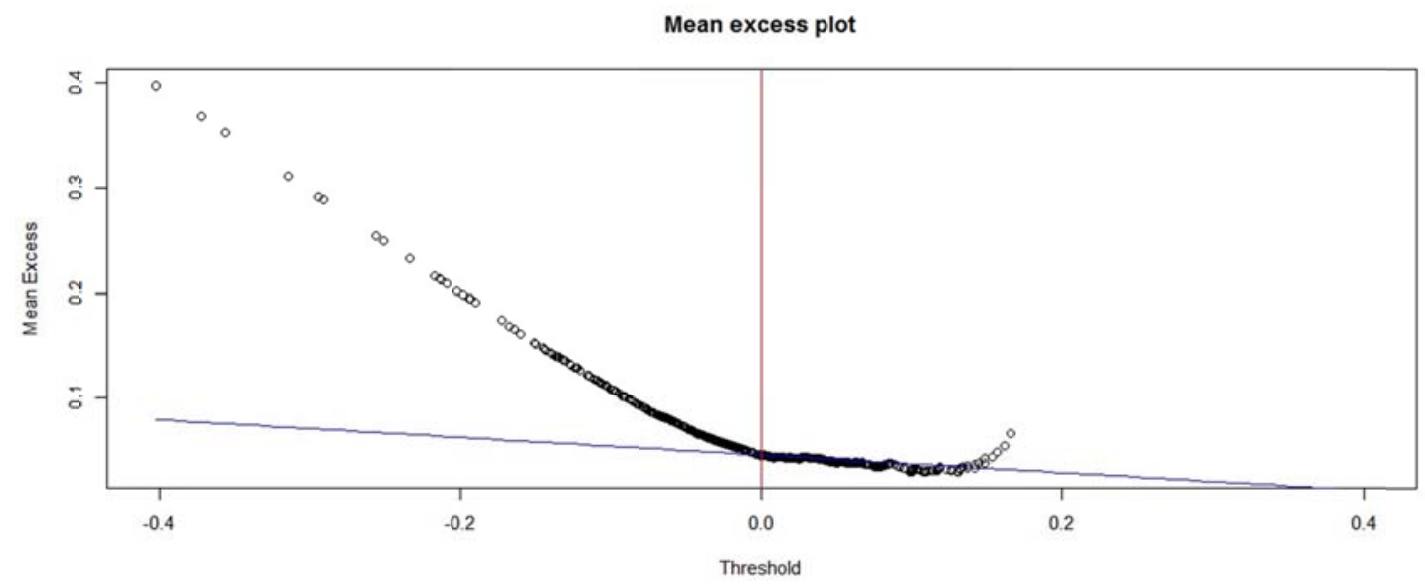

Figure 36. Mean excess plot for the MaidSafeCoin/ USD exchange rate with a vertical line at the threshold $u=0$

Note the two groups which we can detect: MaidSafeCoin and Monero show similar characteristics. The second group consists of Bitcoin, Litecoin, Dash, Ripple.

\subsection{Cryptocurrencies and the Generalized Extreme Value Distribution}

In probability theory and statistics, the generalized extreme value (GEV) distribution is a family of continuous probability distributions developed within extreme value theory to combine the Gumbel, Fréchet and Weibull families also known as type I, II and III extreme value distributions. The Fisher-Tippett-Gnedenko theorem is a general result in extreme value theory regarding asymptotic distribution of extreme order statistics. The maximum of a sample of iid random variables after proper renormalization can only converge in distribution to one of three possible distributions, the Gumbel distribution, the Fréchet distribution, or the Weibull distribution. Credit for the extreme value theorem (or convergence to types theorem) is given to (Gnedenko, 1948).

The role of the extremal types theorem for maxima is similar to that of the central limit theorem for averages, except that the central limit theorem applies to the average of a sample from any distribution with finite variance, while the Fisher-Tippet-Gnedenko theorem only states that if the distribution of a normalized maximum converges, then the limit has to be one of a particular class of distributions. It does not state that the distribution of the normalized maximum does converge. The existence of a limit distribution requires regularity conditions on the tail of the distribution. Despite this, the GEV distribution is often used as an approximation to model the maxima of long (finite) sequences of random variables.

Theorem 3 (Fisher-Tippet-Gnedenko). Let $\left(X_{1}, X_{2}, \ldots, X_{n}\right)$ be a sequence of independent and identically distributed random variables, and $M_{n}=\max \left\{X_{1}, \ldots, X_{n}\right\}$. If a sequence of pairs of real numbers $\left(a_{n}, b_{n}\right)$ exists such that each $a_{n}>0$ and $\lim _{n \rightarrow \infty} P\left(\frac{M_{n}-b_{n}}{a_{n}} \leq x\right)=F(x)$ where $F$ is a non-degenerate distribution function, then the limit distribution $F$ belongs to 
either the Gumbel, the Fréchet or the Weibull family. These can be grouped into the generalized extreme value distribution.

By the extreme value theorem the GEV distribution is the only possible limit distribution of properly normalized maxima of a sequence of independent and identically distributed random variables.

Definition 7 (Generalized extreme value distribution).The generalized extreme value $d$ istribution has cumulative distribution function

$$
F_{(\mu, \sigma, \xi)}(x)=\left\{\begin{array}{l}
\exp \left\{-\left[1+\xi\left(\frac{x-\mu}{\sigma}\right)\right]^{-\frac{1}{\xi}}\right\} \text { for } \xi \neq 0 \\
\exp \left(-\exp \left(-\frac{x-\mu}{\sigma}\right)\right) \text { for } \xi=0
\end{array}\right.
$$

for $1+\xi(x-\mu) / \sigma>0$, where $\mu \in \mathbb{R}$ is the location parameter, $\sigma>0$ the scale parameter and $\xi \in \mathbb{R}$ the shape parameter. For $\xi>0$, the support is $x>\mu-\sigma / \xi$, while for $\xi<0$, it is $x<\mu-\sigma / \xi$. For $\xi=0, x \in \mathbb{R}$.

We are fitting a GEV distribution to the block maxima of the negative of our cryptocurrency exchange rates versus the USD. The block maxima are taken over periods of one month. The estimated parameters, together with their standard errors, as well as the negative log-likelihood is given in Table 17. The estimation procedure is using the maximum likelihood method.with a block size of one month of observations.

Table 17. Maximum likelihood t of generalized extreme value distribution

\begin{tabular}{|l|c|c|c|l|l|l|l|}
\hline Exchange rate & $\xi$ & $\sigma$ & $\mu$ & $\xi$ s.e. & $\sigma$ s.e. & $\mu$ s.e. & nllh \\
\hline Bitcoin/USD & 0.248 & 0.029 & 0.050 & 0.289 & 0.006 & 0.007 & -51 \\
\hline Dash/USD & 0.236 & 0.034 & 0.079 & 0.196 & 0.006 & 0.008 & -46 \\
\hline Litecoin/USD & 0.326 & 0.036 & 0.062 & 0.182 & 0.007 & 0.008 & -43 \\
\hline Maid/USD & 0.124 & 0.029 & 0.112 & 0.140 & 0.005 & 0.006 & -53 \\
\hline Monero/USD & 0.003 & 0.041 & 0.112 & 0.147 & 0.006 & 0.009 & -45 \\
\hline Ripple/USD & 0.231 & 0.044 & 0.084 & 0.205 & 0.008 & 0.010 & -40 \\
\hline
\end{tabular}

Litecoin has the highest $\xi$ parameter of all cryptocurrencies. We show the density of a GEV distribution with parameters $\xi=0.326, \sigma=0.036$ and $\mu=0.062$ (Litecoin/ USD, Figure 39) versus the parameters $\xi=0.003, \sigma=0.041$ and $\mu=0.112$ (Monero/ USD, Figure 41) in the following chart so that the difference between the Litecoin/ USD exchange rate and Monero/ USD becomes obvious from a graphical point of view. 


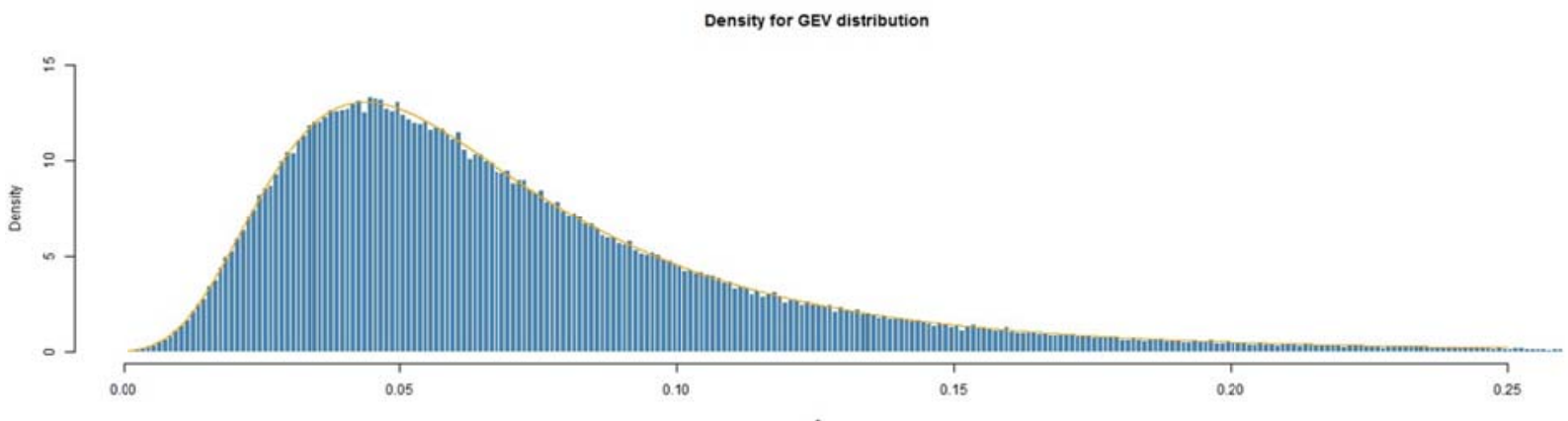

Figure 37. Density of a GEV distribution (Bitcoin/USD) with parameters $\xi=0.248$, $\sigma=0.029$ and $\mu=0.050$

Density for GEV distribution

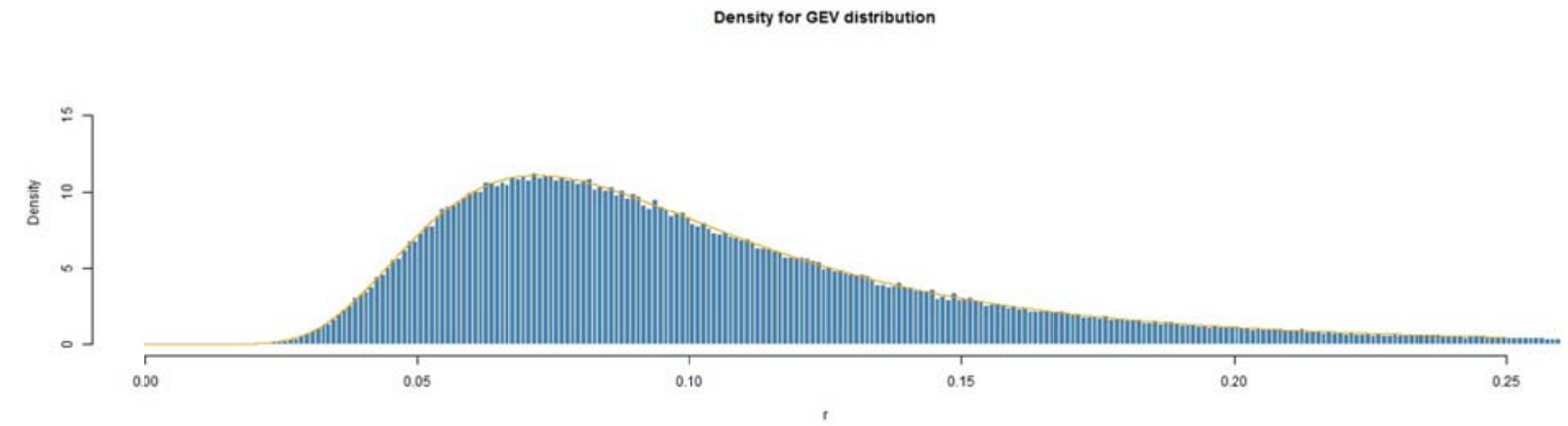

Figure 38. Density of a GEV distribution (Dash/USD) with parameters $\xi=0.236$, $\sigma=0.034$ and $\mu=0.079$

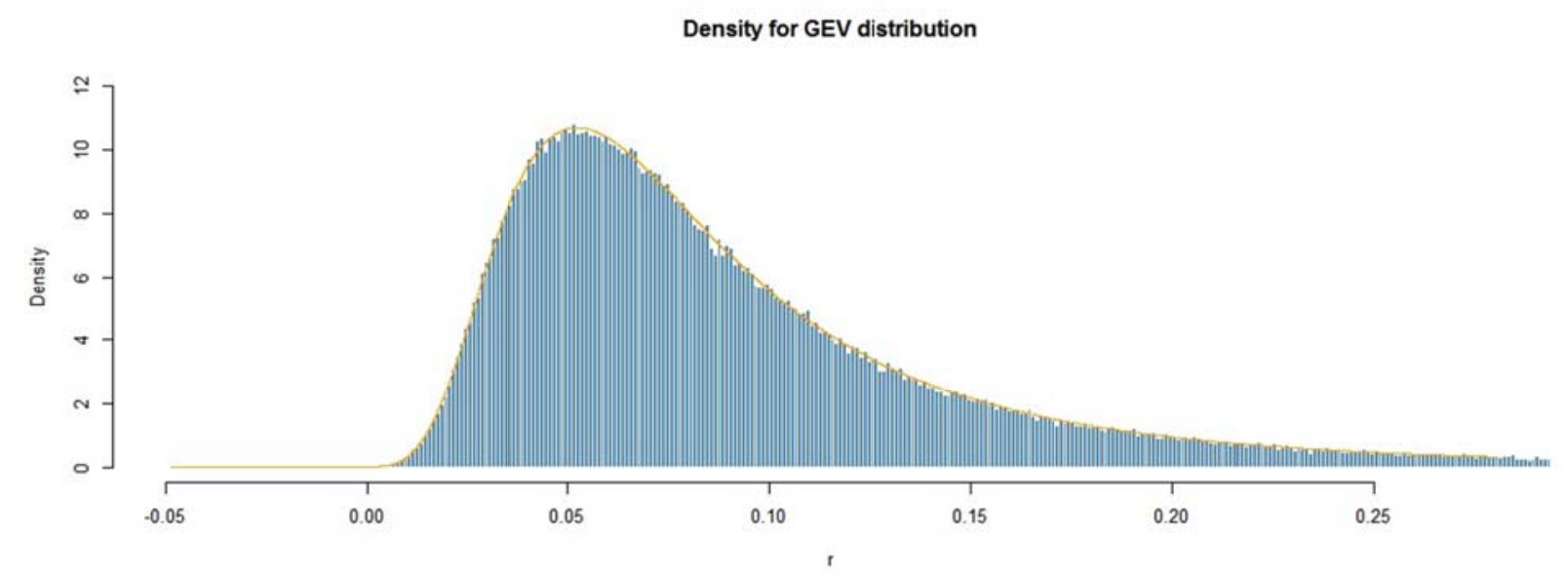

Figure 39. Density of a GEV distribution (Litecoin/USD) with parameters $\xi=0.326$, $\sigma=0.036$ and $\mu=0.062$ 
Density for GEV distribution

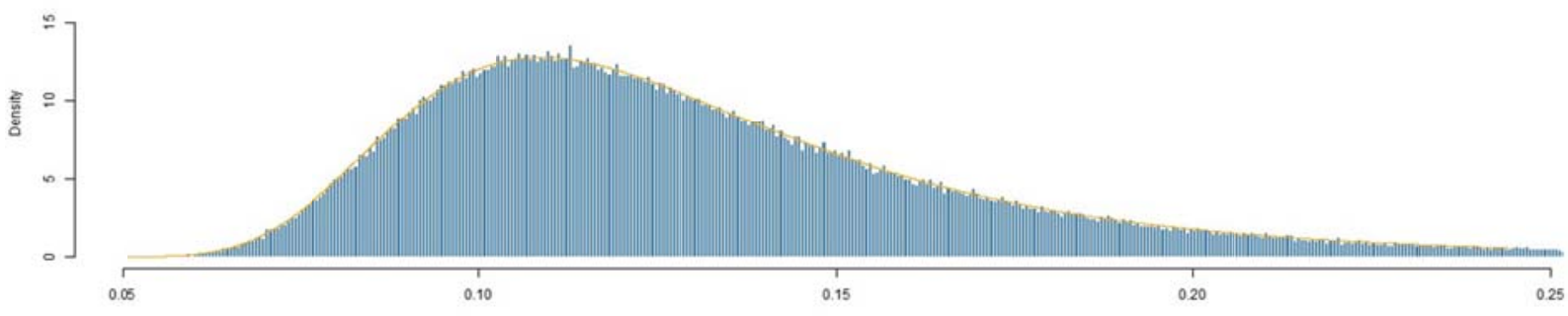

Figure 40. Density of a GEV distribution (Maid/USD) with parameters $\xi=0.124$,

$$
\sigma=0.029 \text { and } \mu=0.112
$$

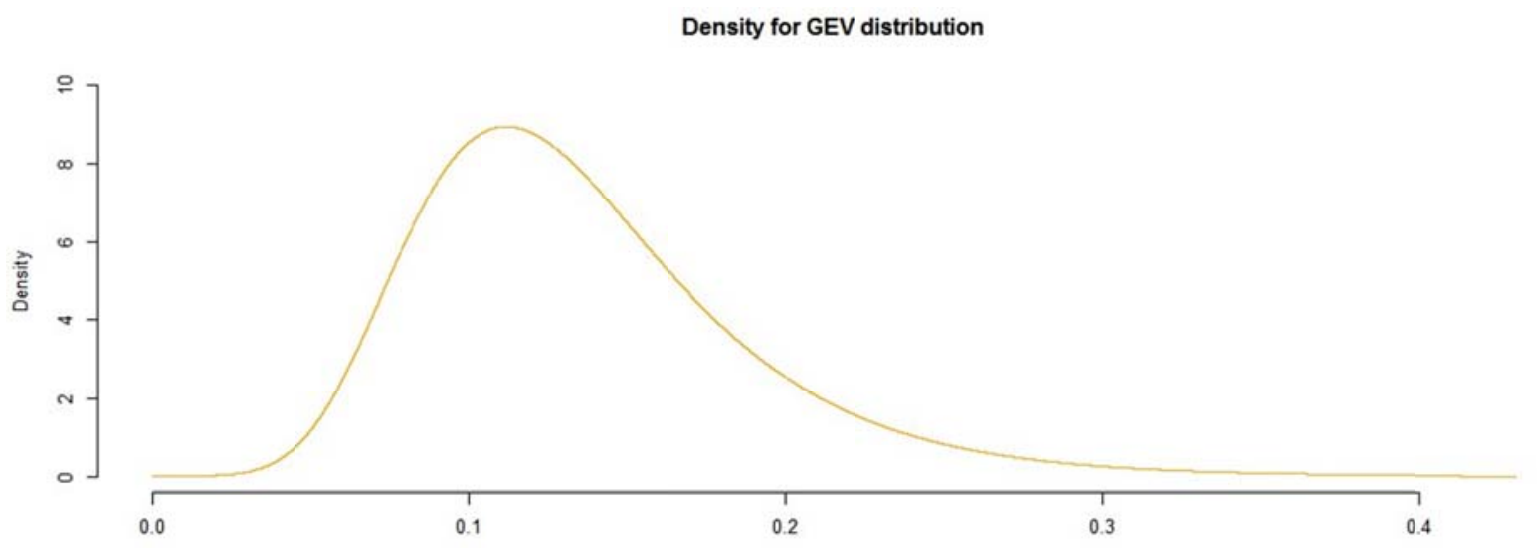

Figure 41. Density of a GEV distribution (Monero/USD) with parameters $\xi=0.003$,

$$
\sigma=0.041 \text { and } \mu=0.112
$$

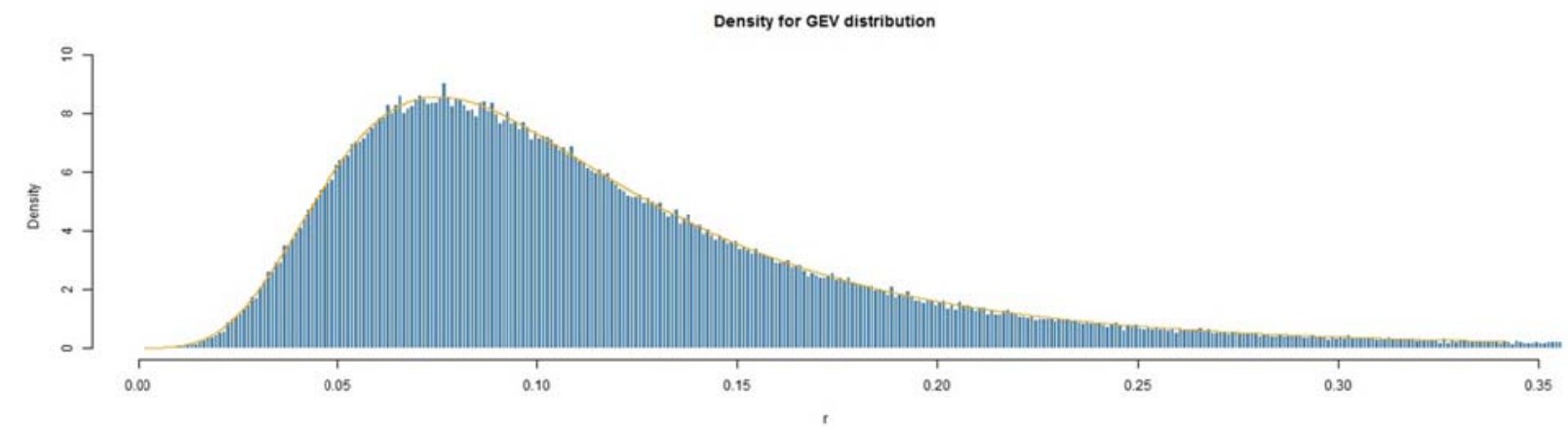

Figure 42. Density of a GEV distribution (Ripple/ USD) with parameters $\xi=0.231$, $\sigma=0.044$ and $\mu=0.084$

By comparing Figures 39 and 41, and looking at the scales of the x-axis and the y-axis, we see that Monero is riskier than Litecoin, despite having the lower $\xi$ parameter. The higher 
value for $\mu$ for Monero leads to this higher risk.

\section{Conclusion}

Cryptocurrencies became popular in 2009 with the emergence of Bitcoin. Since then, cryptocurrencies have shown an unprecedented growth, and are widely available in the public view since at least 2013. We have analyzed the risks of the six most important ones of them, Bitcoin, Litecoin, Dash, Monero, MaidSafeCoin, Ripple. It is important for regulators and central banks world-wide as well as investors, both institutional ones and retail clients, to have a full understanding of the underlying risks of those currencies. By taking data from June 2014 until September 2016, we could show that cryptocurrency returns are extremely volatile, much riskier and exhibit heavier tail behaviour than the traditional fiat currencies.

To our knowledge, this is the first study looking at the statistical properties and extreme value behaviour of cryptocurrencies. The annualized volatility of our cryptocurrencies is substantially larger than any of the standard financial assets, such as the fiat currencies, equities or commodities, with the annual volatility reaching levels beyond $100 \%$. Volatility over time shows large swings and is very unstable, with some of return volatilities regularly reaching very high levels. Our cryptocurrencies are mildly correlated, except for Bitcoin and Litecoin which are very highly correlated. Except for the second half of 2015, Bitcoin/Litecoin generally show a correlation above 70\%. Ripple shows the lowest correlation to all the other currencies. In order to study dependence we are using copulas. Both the empirical and the Gaussian copula are fitted to the data. The tail dependence coefficient is computed both with a parametric and a non-parametric method. Value-at-risk and expected shortfall, as the most common risk measures, show that you can expect a loss of more than $10 \%$ once every 20 days, with the loss on such a day then reaching levels of up to $20 \%$. Again, this risk is much higher than one would expect in one of the traditional asset classes. Using the extremal index as a measure of clustering of extreme events, our currency returns exhibit clear clustering behaviour. In other words, you expect that extreme events are likely to happen on consecutive days, another element of a risky asset. Lastly, we have fitted the GPD and the GEV distributions to exceedances over a threshold and monthly maxima returns, respectively. Bitcoin, Litecoin and Ripple show similar, and very risky, behaviour. Using the GEV distribution, Bitcoin, Litecoin, Ripple and Dash show similar and the riskiest returns. The investment in cryptocurrencies is highly speculative and the risk of losing a lot if not all of the investment is very high. In contrast to typical fiat currencies, cryptocurrencies are not controlled by an entity like a government or a bank. Opinions about an investment in cryptocurrencies are divided between supporters which point toward the growing usage, while conservative investors rather see it as a speculative bubble. The inwestment in Bitcoin in general can be considered safer compared to other cryptocurrencies due the higher liquidity and the lowest annual volatility of $62 \%$ compared to the other five cryptocurrencies above.

There are various ways for future research in that area. We have been using aggregate cryptocurrency indices from multiple exchanges. It is worthwhile understanding the different market places and how the prices differ from each other. Furthermore, we have noted that 
Bitcoin and Litecoin show similar statistical properties. This is partly based on the same technical foundations. However, a thorough study of why the returns have such a high correlation needs to be undertaken.

To summarize we have shown that cryptocurrencies show risk characteristics that go above and beyond any of the risks that we observe in traditional asset classes. Bitcoin, as the most mature and largest cryptocurrency, is still one of the less risky cryptocurrencies. Any investor in cryptocurrencies needs to be aware of those risks.

\section{References}

Alexander, C., \& Lazar, E. (2006). Normal mixture garch $(1,1)$ : Applications to exchange rate modelling. Journal of Applied Econometrics, 21(3), 307-336. https://doi.org/10.1002/jae.849

Artzner, P., Delbaen, F., Eber, J. M., \& Heath, D. (1999). Coherent measures of risk. Mathematical Finance, 9(3), 203-228. https://doi.org/10.1111/1467-9965.00068

Balkema, A. A., \& Haan de, L. (1974). Residual life time at great age. The Annals of Probability, 2(5), 792-804. http://dx.doi:10.1214/aop/1176996548

Bauer, C. (2000). Value at risk using hyperbolic distributions. Journal of Economics and Business, 52(5), 455-467. https://doi.org/10.1016/S0148-6195(00)00026-6

Chok, N. S. (2010). Pearson's versus Spearman's and Kendall's correlation coefficients for continuous data. Ph. D. thesis, University of Pittsburgh.

Chu, J., Nadarajah, S., \& Chan, S. (2015). Statistical analysis of the exchange rate of bitcoin. PLOS ONE, 10(7), e0133678. https://doi.org/10.1371/journal.pone.0133678

CoinMarketCap. (2016). Crypto-currency market capitalizations. Online [Available]: https://coinmarketcap.com/

Coles, S. (2001). An introduction to statistical modeling of extreme values (2nd ed.). London: Springer-Verlag New York. https://doi.org/10.1007/978-1-4471-3675-0

Coppes, R. C. (1995). Are exchange rate changes normally distributed? Economics Letters, 47(2), 117-121. https://doi.org/10.1016/0165-1765(94)00571-I

Corlu, C. G., \& Corlu, A. (2015.) Modelling exchange rate returns: which flexible distribution to use? Quantitative Finance, 15(11), 1851-1864. https://doi.org/10.1080/14697688.2014.942231

Ferro, C. A. T., \& Segers, J. (2003). Inference for clusters of extreme values. Journal of the Royal Statistical Society: Series B (Statistical Methodology), 65(2), 545-556. http://dx.doi.org/10.1111/1467-9868.00401

Gandal, N., \& Halaburda, H. (2016). Can we predict the winner in a market with network effects?Competition in cryptocurrency market. Games, 7(3), http://dx.doi.org/10.3390/g7030016 


\section{Macrothink}

International Finance and Banking ISSN 2374-2089 2017, Vol. 4, No. 1

Gnedenko, B. V. (1948). On a local limit theorem of the theory of probability. Uspekhi Mat. Nauk, 3(25), 187-194.

Gurrola, P. (2007). Capturing fat-tail risk in exchange rate returns using su curves: A comparison with the normalmixture and skewed student distributions. The Journal of Risk, 10(2), 73. https://doi.org/10.21314/JOR.2007.163

Hosking, J. R. M., \& Wallis, J. R. (1987). Parameter and quantile estimation for the $\begin{array}{llll}\text { generalized } & \text { pareto } & \text { distribution. } & \text { Technometrics, }\end{array}$ http://dx.doi.org/10.2307/1269343

Jaworski, P., Durante, F., \& Hardle, W. K. (2010). Copula theory and its applications: Proceedings of the workshop held in Warsaw, 25-26 September 2009. Heidelberg: Springer-Verlag Berlin and Heidelberg GmbH \& Co. K. https://doi.org/10.1007/978-3-642-12465-5

Kilic, R. (2007). Conditional volatility and distribution of exchange rates: Garch and figarch models with nigdistribution. Studies in Nonlinear Dynamics \& Econometrïcs, 11(3).

Linden, M. (2005). Estimating the distribution of volatility of realized stock returns and exchange rate changes. Physica A: Statistical Mechanics and its Applications, 352(2), 573-583. https://doi.org/10.1016/j.physa.2004.12.024

Lopez, H. F., Rodriguez, B. D., \& Ortiz, A. F. (2011). The stochastic volatility of the peso-dollarexchange rate: The floating regime in Mexico. Investigacion Economica, 70(276), 19.

Nadarajah, S., Afuecheta, E., \& Chan, S. (2015). A note on modelling exchange rate returns: Which flexible distribution to use? Quantitative Finance, 15(11), 1777-1785. https://doi.org/10.1080/14697688.2015.1032997

Nakajima, J. (2013). Stochastic volatility model with regime-switching skewness in heavy-tailed errors for exchangerate returns. Studies in Nonlinear Dynamics and Econometrics, 17(5), 499-520.

Osterrieder, J. (2016). The Statistics of Bitcoin and Cryptocurrencies. [Online] Available: https://ssrn.com/abstract $=2872158$

Osterrieder, J., \& Lorenz, J. (2016). A Statistical Risk Assessment of Bitcoin and Its Extreme Tail Behaviour. [Online] Available: https://ssrn.com/abstract=2867339

Osterrieder, J., Lorenz, J., \& Strika, M. (2016). Bitcoin and Cryptocurrencies-Not for the Faint-Hearted. Advanced Risk \& Portfolio Management Paper. [Online] Available: https://ssrn.com/abstract $=2867671$

Pickands, J. (1975). Statistical inference using extreme order statistics. The Annals of Statistics, 3(1), 119-131. https://doi.org/10.1214/aos/1176343003

Pipien, M. (2004). Garch processes with skewed-t and stable conditional distributions. bayesian analysis forpln/usd exchange rate. Folia Oeconomica Cracoviensia, 45, 45-62. 
Schmid, F., \& Schmidt, R. (2007). Multivariate conditional versions of spearman's rho and related measures of tail dependence. Journal of Multivariate Analysis, 98(6), 1123-1140. http://dx.doi.org/10.1016/j.jmva.2006.05.005

Sheskin, D. J. (2007). Handbook of Parametric and Nonparametric Statistical Procedures (4th ed.). UK: Chapman \& Hall/CRC.

\section{Copyright Disclaimer}

Copyright for this article is retained by the author(s), with first publication rights granted to the journal.

This is an open-access article distributed under the terms and conditions of the Creative Commons Attribution license (http://creativecommons.org/licenses/by/3.0/). 